\title{
Power Allocation and Spectrum Sharing in Wireless Networks: An Implementation Theory Approach
}

Ali Kakhbod, Ashutosh Nayyar, Shrutivandana Sharma and Demosthenis Teneketzis 



\section{Introduction}

Today's communication networks provide a large number of services (e.g. telephone connections, live audio and video broadcasting, email, Internet etc.), each with its own quality of service requirements, to an increasingly large number of users. All these services are provided via the network's infrastructure that contains a limited number of resources. Thus, efficient allocation of the network's resources to its users is of great importance as it can have a significant impact on network performance.

Unlike traditional communication networks where users are assumed to cooperate with the network and accept the resource allocation decisions made by the network manager/service provider, the modern communication technology such as cognitive radio has enabled users to make intelligent decisions and influence the resource allocation so as to maximize their own benefit. This poses a challenge to efficient resource allocation because it not only needs to adapt with decentralized information about different users' needs and preferences, but also needs to take into account the users' strategic behavior.

In recent years game theoretic approaches have evolved to investigate strategic behavior of users in communication networks and their impact on resource allocation and network performance. However, game theoretic methodology restricts itself to analyzing the effects of users' strategic interactions under specific/given scenarios (e.g. given valuation functions and given price rules for the users). In general, because of users' conflicting objectives, their strategic interactions do not result in desirable network performance. In order to achieve desirable performance, external incentives must be provided to the users that align their individual objectives with the network-wide performance objective. The design of such incentives must address the following challenges: (1) It must be based on the information revealed by the network users; and (2) It must anticipate users' strategic behavior in their revelation of information according to the created incentives.

Mechanism design provides a methodology to design appropriate incentives that address the above challenges and help to achieve various performance objectives such as, maximizing the social welfare generated by the use of network resources or maximizing the revenue collected by the network owner. In this chapter, we introduce the concepts of implementation theory, a component of the theory of mechanism design. Based on these concepts we present an approach for designing decentralized mechanisms for power and spectrum allocation problems that arise in wireless communication networks.

\section{Chapter Organization}

The chapter is organized as follows. In Section 2 we present a brief overview of implementation theory concepts that are used in this chapter. In Section 3 , we present an approach to power allocation and spectrum sharing problems 
when the mechanism designer's objective is social welfare maximization or Pareto optimality. In Section 4, we consider problems of revenue maximization. We conclude in Section 5 with a critique of the implementation theory approach and results, and a discussion of important open issues in power allocation and spectrum sharing problems.

\section{What is Implementation Theory?}

Implementation theory is a component of the modern economic theory of mechanism design. Implementation theory provides an analytical framework for situations where resources have to be allocated among agents/users but the information needed to make these allocation decisions is dispersed and privately held, and the agents/users possessing the private information behave strategically and are self-utility maximizers.

In any situation where the information needed to make decisions is dispersed, it is necessary to have information exchange among the agents/users possessing the information. Allocation decisions are made after the information exchange process terminates. These decisions must optimize some pre-specified performance metric. The objectives of implementation theory are: (1) To determine, for any given performance metric, whether or not there exists an information exchange process and an allocation rule that achieve optimal allocations with respect to that metric when the users are strategic. (2) To determine systematic methodologies for designing information exchange processes and allocation rules that achieve optimal allocations with respect to performance metrics for which the answer to (1) is positive. (3) To determine alternative criteria for the design of information exchange processes and allocation rules that lead to "satisfactory" allocations, with respect to performance metrics for which the answer to (1) is negative.

The key concept in the development of implementation theory is that of game form or mechanism. A game form/mechanism consists of two components: (1) a message/strategy space, that is, a communication alphabet through which the agents/users exchange information with one another. (2) an allocation rule (called outcome function) that determines the allocations after the communication and information exchange process terminates. Most mechanisms employ monetary incentives and payments to achieve desirable resource allocations. In such cases, the outcome function specifies the resource allocations as well as the monetary incentives and payments.

A game form along with the agents'/users' utilities defines a game. The allocations made (through the outcome function) at the equilibria of the game determine the result of the decentralized allocation problem. The key objectives in the design of a game form/mechanism are: 
1 To provide incentive to the strategic agents/users so that they prefer to participate in the allocation process rather than abstain from it.

2 To obtain at one, or preferably at all, equilibria of the game induced by the mechanism, allocations that are optimal with respect to some pre-specified performance criterion. For example, it may be desired that the allocations obtained by the game form/mechanism are the same as those obtained by the solution of the corresponding centralized allocation problem.

3 To obtain a balanced budget at all equilibria of the game induced by the mechanism. That is, at all equilibria, the money received by some of the system's agents/users as part of the incentives provided by the mechanism must be equal to the money paid by the rest of the agents/users.

4 To minimize the complexity of the mechanism (measured, for example, by the dimensionality of the message space as well as by its computational complexity).

In the rest of this section we will formally present the key ideas, objectives and results of implementation theory that are relevant to the topics of this chapter.

\section{Game Forms/Mechanisms}

Resource allocation problems can be described by the following triple: $(\mathcal{E}, \mathcal{A}, \gamma)$. $\mathcal{E}$ describes the set of all possible environments/instances of the problem. An $e \in \mathcal{E}$ specifies the agents' individual endowments, resource constraints, utilities etc. The environment is defined as the set of circumstances that can not be changed by the designer of the game form/mechanism or by the agents. The set $\mathcal{A}$ is the outcome space. It is the set of all possible allocations (e.g. resource exchanges, monetary payments) made to the system's agents. The rule $\gamma$, is called social choice rule/social choice correspondence/ goal correspondence. The rule $\gamma: \mathcal{E} \mapsto \mathcal{A}$ assigns to every $e \in \mathcal{E}$, a set $\gamma(e) \subset \mathcal{A} ; \gamma(e)$ is the choice set for the instance of the resource allocation problem corresponding to $e$.

The above setup describes a centralized resource allocation problem. It corresponds to the case where one of the system's agents has enough information about the environment so as to determine the outcomes that satisfy the goal correspondence $\gamma$. Generally this is not the case. Usually, different agents have different information about the environment. For this reason it is desired to devise a mechanism for information exchange and resource allocation that leads, for every instance $e$ of the resource allocation problem, to an allocation in $\gamma(e)$.

When the system's agents are strategic and self-utility maximizers the resource allocation mechanism is described by a game form. To formally define a game form, consider a system consisting of $N$ agents/users. Let $\mathcal{N}:=\{1,2, \ldots, N\}$ be the set of agents. The game form is defined as a pair $(\mathcal{M}, h)$, where $\mathcal{M}=\Pi_{i=1}^{N} \mathcal{M}_{i}$, $\mathcal{M}_{i}$ is the strategy/message space of agent $i$ and $h: \mathcal{M} \mapsto \mathcal{A}$ is the outcome function. Thus, for each message/strategy profile $m:=\left(m_{1}, m_{2}, \ldots, m_{N}\right) \in \mathcal{M}$, 
$h(m) \in \mathcal{A}$ represents the resulting outcome in $\mathcal{A}$. The game form operates in 3 steps:

1 The mechanism designer announces the pair $(\mathcal{M}, h)$.

2 An instance $e \in \mathcal{E}$ of the environment is realized. The realization of environment $e$ specifies, among other things, the utilities $u_{i}, i \in \mathcal{N}$ of all agents. Depending on its utilities and the specified mechanism, each agent decides whether or not to participate in the mechanism. The agents who reject the mechanism get some exogenously specified "reservation utility", which is usually a number independent of the environment $e$; we set this number to be zero.

3 The agents who choose to participate in the mechanism play the game induced by the mechanism. In this game, $\mathcal{M}_{i}$ is the strategy space of player $i$, and for every strategy profile $m \in \mathcal{M}, u_{i}(h(m))$ is the utility payoff of player $i$. We denote this game by $(\mathcal{M}, h, e)$

The mechanism designer is interested in the outcomes that occur at various equilibria of the game induced by the game form. In the next section, we discuss different notions of implementation depending on the nature of the game form and the requirements of the mechanism designer.

\section{Implementation in Different Types of Equilibria}

An equilibrium concept for a game is a prediction of the strategic behavior of the agents playing that game. For a given game form, $(\mathcal{M}, h)$, an equilibrium concept establishes a correspondence between the space of agents' environment and the message space. That is, an equilibrium concept establishes a correspondence $\bar{\Lambda}$ that identifies a subset $\bar{\Lambda}(\mathcal{M}, h, e)$ of $\mathcal{M}$ as the expected message profiles of the agents. For $m \in \bar{\Lambda}(\mathcal{M}, h, e), h(m)$ is the resulting outcomes of the game. We define $\mathcal{A}_{\bar{\Lambda}} \subset \mathcal{A}$ as the set of outcomes associated with all the $\bar{\Lambda}$-type equilibria of the game $(\mathcal{M}, h, e)$,

$$
\mathcal{A}_{\bar{\Lambda}}:=\mathcal{A}_{\bar{\Lambda}}(\mathcal{M}, h, e):=\{a \in \mathcal{A} \mid \exists m \in \bar{\Lambda}(\mathcal{M}, h, e) \text { s.t. } h(m)=a\}
$$

Definition 1 A social choice rule/ social choice correspondence $\gamma: \mathcal{E} \mapsto \mathcal{A}$ is said to be implementable (respectively, weakly implementable) in the equilibrium concept $\bar{\Lambda}$ if there is a game form $(\mathcal{M}, h)$ such that $\mathcal{A}_{\bar{\Lambda}}(\mathcal{M}, h, e)=\gamma(e)$ (respectively, $\left.\mathcal{A}_{\bar{\Lambda}}(\mathcal{M}, h, e) \subset \gamma(e)\right)$ for all $e \in \mathcal{E}$. The game form $(\mathcal{M}, h)$ is said to implement (respectively, weakly implement) the social choice rule $\gamma$ in $\bar{\Lambda}$.

A comparison between implementation and weak implementation appears in (Thompson 2001). We now describe several possible choices of the equilibrium concept used in implementation theory.

\subsubsection{Dominant Strategy Equilibrium}

The most desirable equilibrium concept is that of a dominant strategy equilibrium. The existence of a dominant strategy $m^{*}:=\left(m_{1}^{*}, m_{2}^{*}, \ldots, m_{N}^{*}\right)$ for game 
$(\mathcal{M}, h, e)$ implies that for every agent $i \in \mathcal{N}$, the choice $m_{i}^{*}$ maximizes agent $i$ 's utility function irrespective of the other agents' choice of strategies. Formally,

Definition 2 A strategy profile $m^{*}:=\left(m_{1}^{*}, m_{2}^{*}, \ldots, m^{*} N\right) \in \mathcal{M}$ is a dominant strategy equilibrium for the game $(\mathcal{M}, h, e)$ if for all $i \in \mathcal{N}$,

$$
u_{i}\left(h\left(m_{i}^{*}, m_{-i}\right)\right) \geq u_{i}\left(h\left(m_{i}, m_{-i}\right)\right)
$$

for all $m_{i} \in \mathcal{M}_{i}$, for all $m_{-i}:=\left(m_{1}, m_{2}, \ldots, m_{i-1}, m_{i+1}, \ldots, m_{N}\right) \in \Pi_{j \neq i} \mathcal{M}_{j}$; where $u_{i}, i \in \mathcal{N}$ are the utility functions of the agents under the realization $e$ of the environment.

Let $D(\mathcal{M}, h, e)$ be the set of dominant strategy equilbria of the game $(\mathcal{M}, h, e)$ and

$$
\mathcal{A}_{D}:=\mathcal{A}_{D}(\mathcal{M}, h, e):=\{a \in \mathcal{A} \mid \exists m \in D(\mathcal{M}, h, e) \text { s.t. } h(m)=a\}
$$

be the set of outcomes associated with all the dominant strategy equilibria of the game $(\mathcal{M}, h, e)$. The game form $(\mathcal{M}, h)$ implements (respectively, weakly implements) a social choice correspondence $\gamma$ in dominant strategy equilibrium if

$$
\mathcal{A}_{D}(\mathcal{M}, h, e)=\gamma(e)
$$

(respectively, $\left.\mathcal{A}_{D}(\mathcal{M}, h, e) \subset \gamma(e)\right)$ for all $e \in \mathcal{E}$.

\subsubsection{Nash Equilibrium}

For any given game $(\mathcal{M}, h, e)$, a pure Nash equilibrium (NE) is a message/strategy profile $m^{*}:=\left(m_{1}^{*}, m_{2}^{*}, \ldots, m_{N}^{*}\right) \in \mathcal{M}$ such that for all $i \in \mathcal{N}$,

$$
u_{i}\left(h\left(m_{i}^{*}, m_{-i}^{*}\right)\right) \geq u_{i}\left(h\left(m_{i}, m_{-i}^{*}\right)\right),
$$

for all $m_{i} \in \mathcal{M}_{i}$, where $m_{-i}^{*}:=\left(m_{1}^{*}, m_{2}^{*}, \ldots, m_{i-1}^{*}, m_{i+1}^{*}, \ldots, m_{N}^{*}\right)$ and $u_{i}, i \in \mathcal{N}$ are the utility functions of the agents under the realization $e$ of the environment. Let $N E(\mathcal{M}, h, e)$ be the set of Nash equilbria of the game $(\mathcal{M}, h, e)$ and

$$
\mathcal{A}_{N E}:=\mathcal{A}_{N E}(\mathcal{M}, h, e):=\{a \in \mathcal{A} \mid \exists m \in N E(\mathcal{M}, h, e) \text { s.t. } h(m)=a\}
$$

The game form $(\mathcal{M}, h)$ implements (respectively, weakly implements) a social choice correspondence $\gamma$ in Nash equilibrium if

$$
\mathcal{A}_{N E}(\mathcal{M}, h, e)=\gamma(e)
$$

(respectively, $\left.\mathcal{A}_{N E}(\mathcal{M}, h, e) \subset \gamma(e)\right)$ for all $e \in \mathcal{E}$.

\subsubsection{Bayesian Nash Equilibrium}

Consider next pure Bayesian Nash equilibrium (BNE) as the equilibrium concept. The environment space is described as a product space $\mathcal{E}=\mathcal{E}_{1} \times \mathcal{E}_{2} \times \ldots \times \mathcal{E}_{N}$. Each agent $i \in \mathcal{N}$ knows its own environment $e_{i} \in \mathcal{E}_{i}$ and has a pdf $p\left(\cdot \mid e_{i}\right)$ on the set $\mathcal{E}_{-i}=\mathcal{E}_{1} \times \mathcal{E}_{2} \times \ldots \mathcal{E}_{i-1} \times \mathcal{E}_{i+1} \times \ldots \mathcal{E}_{N}$ of environments of all other agents $j \neq i$. The functional form of $p\left(\cdot \mid e_{j}\right), j \in \mathcal{N}$ is known by the mechanism 
designer for all $e_{j} \in \mathcal{E}_{j}$, for all $j \in \mathcal{N}$. However, the mechanism designer does not know the realizations, $e_{j}, j \in \mathcal{N}$. A pure BNE is a vector of strategy rules $\left(\sigma_{1}^{*}(\cdot), \sigma_{2}^{*}(\cdot), \ldots, \sigma_{N}^{*}(\cdot)\right)$ where $\sigma_{i}: \mathcal{E}_{i} \mapsto \mathcal{M}_{i}$, which has the following property: For every $e_{i} \in \mathcal{E}_{i}, i \in \mathcal{N}$,

$\int_{\mathcal{E}_{-i}} u_{i}\left(h\left(\sigma_{i}^{*}\left(e_{i}\right), \sigma_{-i}^{*}\left(e_{-i}\right)\right)\right) p\left(e_{-i} \mid e_{i}\right) d e_{-i} \geq \int_{\mathcal{E}_{-i}} u_{i}\left(h\left(\sigma_{i}\left(e_{i}\right), \sigma_{-i}^{*}\left(e_{-i}\right)\right)\right) p\left(e_{-i} \mid e_{i}\right) d e_{-i}$,

for all $\sigma_{i}: \mathcal{E}_{i} \mapsto \mathcal{M}_{i}$, where $\sigma_{-i}^{*}\left(e_{-i}\right):=\left(\sigma_{1}^{*}\left(e_{1}\right), \sigma_{2}^{*}\left(e_{2}\right), \ldots, \sigma_{i-1}^{*}\left(e_{i-1}\right), \sigma_{i+1}^{*}\left(e_{i+1}\right)\right.$, $\left.\ldots, \sigma_{N}^{*}\left(e_{N}\right)\right)$ and $u_{i}, i \in \mathcal{N}$ are the utility functions of the agents under the realization $e_{i}$ of their environment. For a given realization $e$ of the environment, denote by $B N E(\mathcal{M}, h, e)$ the message profiles $m \in \mathcal{M}$ associated with all the vectors of strategy rules that satisfy (0.5). Then, the associated outcomes are

$$
\mathcal{A}_{B N E}:=\mathcal{A}_{B N E}(\mathcal{M}, h, e):=\{a \in \mathcal{A} \mid \exists m \in B N E(\mathcal{M}, h, e) \text { s.t. } h(m)=a\}
$$

The game form $(\mathcal{M}, h)$ implements (respectively, weakly implements) a social choice correspondence $\gamma$ in Nash equilibrium if

$$
\mathcal{A}_{B N E}(\mathcal{M}, h, e)=\gamma(e)
$$

(respectively, $\left.\mathcal{A}_{B N E}(\mathcal{M}, h, e) \subset \gamma(e)\right)$ for all $e \in \mathcal{E}$.

\subsubsection{Which Equilibrium Concept Should the Mechanism Designer} Use?

The equilibrium concept appropriate for a game $(\mathcal{M}, h, e)$ is determined by the information the system's agents possess about the environment (hence, about the game). For example, when every agent has perfect knowledge about every other agent's environment, Nash equilibrium as well as dominant strategy equilibrium are appropriate equilibrium concepts. When every agent only knows its own environment and has a belief, expressed by a probability distribution on the set of environments of all other agents conditioned on its own environment, Bayesian Nash equilibrium and dominant strategy equilibrium are appropriate equilibrium concepts.

\subsubsection{Truthful Implementation}

Game forms/mechanisms $(\mathcal{M}, h)$ where each agent's message space is its environment space, that is, $\mathcal{M}_{i}=\mathcal{E}_{i}$ for all $i \in \mathcal{N}$, are called direct revelation mechanisms. Such mechanisms have a natural appeal and have received significant attention. The interest in direct revelation mechanisms stems from the revelation principle. The revelation principle is the observation that if a game form $(\mathcal{M}, h)$ implements a social choice rule $\gamma: \mathcal{E} \mapsto \mathcal{A}$ in a certain equilibrium concept $\bar{\Lambda}$, then there is a direct revelation mechanism $\left(\mathcal{E}, h^{*}\right)$ which has the following property: (1) Reporting one's true environment is an equilibrium message/strategy of the game induced by $\left(\mathcal{E}, h^{*}\right)$ in the same equilibrium concept $\bar{\Lambda}$; (2) $h^{*}(e) \in \gamma(e)$, for all $e \in \mathcal{E}$. 
Even though the direct revelation mechanism $\left(\mathcal{E}, h^{*}\right)$ has the above property, it does not necessarily implement the social choice rule $\gamma$ in the equilibrium concept $\bar{\Lambda}$. This is because the game induced by the direct revelation mechanism may have multiple equilibria, some of which may give rise to outcomes not contained in $\gamma(e)$ for some $e \in \mathcal{E}$. An example illustrating the above fact appears in (Dasgupta, Hammonds \& Maskin 1979).

Thus, we cannot conclude from the revelation principle that all one ever needs to consider are direct revelation mechanisms. Only under certain conditions a social choice rule can be implemented by a direct revelation mechanism (Dasgupta et al. 1979). Nevertheless, a special concept of implementation associated with direct mechanisms, called truthful implementation, is of interest in some applications (as we will see in Section 4). Truthful implementation in the equilibrium concept $\bar{\Lambda}$ requires that: (R1) For every environment $e \in \mathcal{E}$, truth-telling should be an $\bar{\Lambda}$-equilibrium of the game $\left(\mathcal{E}, h^{*}, e\right)$; (R2) The outcome achieved at the truth-telling equilibrium of the game $\left(\mathcal{E}, h^{*}, e\right)$ should be in the social choice set, that is, $h^{*}(e) \in \gamma(e)$, for all $e \in \mathcal{E}$. Truthful implementation imposes no restrictions on outcomes achieved at any equilibria other than the truth-telling equilibrium.

\section{Desirable Properties of Game Forms}

In addition to implementation in an equilibrium concept, the mechanism designer should try to achieve the other objectives mentioned in Section 2 . We formally define the properties of a mechanism associated with those objectives in this section.

\subsubsection{Individual Rationality}

One of the objectives in the design of a game form is to incentivize all the agents to voluntarily participate in the allocation process under any possible environment. Consider any environment $e \in \mathcal{E}$. If under $e$, agent $i$ decides not to participate, its overall utility is zero (see Section 2.1). If agent $i$ decides to participate in the game induced by the mechanism, its utility is $u_{i}\left(h\left(m^{*}\right)\right)$ where $m^{*}$ is an equilibrium of the game induced by the mechanism. Under $e \in \mathcal{E}$, an agent participates in the game $(\mathcal{M}, h, e)$ if for all equilibria $m^{*}$ of the game, $u_{i}\left(h\left(m^{*}\right)\right) \geq 0$. We can now define individually rational mechanisms as follows:

DEFINITION 3 A mechanism/game form $(\mathcal{M}, h)$ is individually rational if for all $e \in \mathcal{E}$, for all equilibria $m^{*}$ of the game $(\mathcal{M}, h, e)$ and for all $i \in \mathcal{N}$, $u_{i}\left(h\left(m^{*}\right)\right) \geq 0$, where $u_{i}$ is the utility function of agent $i$ in the environment $e$, and 0 is the reservation utility a user receives if it rejects the mechanism (cf. Section 2.1).

\subsubsection{Budget Balance}

Strategic agents are often incentivized to follow the rules of the mechanism through monetary tax and subsidy. Some agents are induced to accept allo- 
cations that may not be their most preferred ones (under the realization $e$ of the environment) by offering them money (subsidy). Conversely, some agents are induced to pay money (tax) for receiving their most preferred allocations. It is desirable that for any environment $e \in \mathcal{E}$, at every equilibrium of the game $(\mathcal{M}, h, e)$ the sum of taxes paid by the agents should be equal to the sum of subsidies received by the agents. Any mechanism $(\mathcal{M}, h)$ that possesses the above property is said to be budget balanced at equilibrium. Budget balance is also desirable off-equilibrium for the following practical reason. Suppose the mechanism designer specifies, along with the mechanism, an iterative message exchange process (tâtonnement process) which for any environment $e \in \mathcal{E}$ is guaranteed to converge to an equilibrium of the game induced by the mechanism. In practice, the message exchange process may terminate when it reaches sufficiently close to the equilibrium (but not the equilbrium). If the mechanism is not budget balanced at these out of equilibrium terminal messages, then possible large amounts of unclaimed money may be left. This is why budget balance is desirable at all out of equilibrium messages.

\subsection{Key Results on Implementation Theory}

We discuss implementation in three types of equilibria: dominant strategy, Nash equilibrium and Bayesian Nash equilibrium.

\subsubsection{Implementation in Dominant Strategies}

We desire implementation of a social choice rule/correspondence in dominant strategies, because in such a situation each agent's optimal strategy is independent of other agents' strategies. Unfortunately, as we discuss below, implementation in dominant strategies is in most cases impossible. This is why we resort to implementation in NE or BNE.

The most general results on implementation in dominant strategies are impossibility results. One of the main results on this topic is the Gibbard-Satterthwaite theorem. It was discovered independently in the 1970s by Gibbard (1973) and Satterthwaite (1975). It is a negative (impossibility) result that has shaped the course of research on implementation theory to a great extent. The theorem shows that for a very general class of problems there is no hope of implementing satisfactory social choice rules in dominant strategies. Specifically, the GibbardSattethwaite theorem states that under certain assumptions on the set of the users' utility functions and the allocation space (see (Mas-Colell, Whinston \& Green 2005, Chapter 23), (Osborne \& Rubinstein 1994, Chapter 10)) a social choice function $\gamma$ is truthfully implementable in dominant strategies if and only if it is dictatorial (see (Mas-Colell et al. 2005, Chapter 21) for the definition of a dictatorial choice function).

Given the negative conclusion of the Gibbard Satterthwaite theorem, if we are to have any hope of implementing desirable social choice rules, we must either consider implementation in a less robust equilibrium concept (e.g. NE, 
$\mathrm{BNE}$ ), or we must focus on more restricted environments $\mathcal{E}$. In the remainder of this section we present positive results on the implementation of desirable social choice rules in dominant strategies when the users'/agents' utilities are quasilinear. Afterwards, in sections 2.4.2 and 2.4.3 we present key results on implementation in NE and BNE, respectively.

Quasilinear utilities have the form

$$
u_{i}\left(\alpha_{i}\right)=v_{i}\left(x_{i}\right)-t_{i}, \quad i \in \mathcal{N}, \quad \text { where, } \quad \alpha_{i}:=\left(x_{i}, t_{i}\right), i \in \mathcal{N},
$$

$v_{i}$ is specified by the realization of the environment, $x_{i}$ denotes the allocation to agent $i$, and $t_{i} \in \mathbb{R}$ denotes the transfer of money to agent $i ; t_{i}>0$ implies that agent $i$ pays a tax, whereas $t_{i}<0$ implies that agent $i$ receives a subsidy. For the class of environments where the users'/agents' utilities are quasilinear we have the following positive result.

THEOREM 1 ((Mas-Colell et al. 2005)) Assume that the users'/agents' utilities are quasilinear and let, for every $e \in \mathcal{E},\left(x_{1}^{*}(e), x_{2}^{*}(e), \cdots, x_{N}^{*}(e)\right)$ be an allocation such that

$$
\sum_{i=1}^{N} v_{i}\left(x_{i}^{*}(e)\right) \geq \sum_{i=1}^{N} v_{i}\left(x_{i}\right)
$$

for any other feasible allocation $\left(x_{1}, \cdots, x_{N}\right) \in \mathcal{A}$. The social choice rule $\gamma$ : $\mathcal{E} \rightarrow \mathcal{A}$, which for each $e \in \mathcal{E}$ selects allocations satisfying (0.7), is truthfully implementable in dominant strategies if for all $i \in \mathcal{N}$,

$$
t_{i}^{*}(e)=-\sum_{j \neq i} v_{j}\left(x_{j}^{*}(e)\right)-\hat{t}_{i}\left(e_{-i}\right),
$$

where $\hat{t}_{i}\left(e_{-i}\right)$ is an arbitrary function of $e_{-i}, e_{-i}:=\left(e_{1}, e_{2}, \cdots, e_{i-1}, e_{i+1}, \cdots, e_{N}\right)$.

A direct revelation mechanism $\left(\mathcal{E}, h^{*}\right)$ where $h^{*}(e)=\left(x_{1}^{*}(e), x_{2}^{*}(e), \cdots, x_{N}^{*}(e)\right.$, $\left.t_{1}^{*}(e), \cdots, t_{N}^{*}(e)\right), x_{i}^{*}(e), i \in \mathcal{N}$, satisfy $(0.7)$ and $t_{i}^{*}(e), i \in \mathcal{N}$, satisfy $(0.8)$ is called a Vickrey-Clark-Groves (VCG) mechanism. A special case of the VCG mechanism is the pivotal mechanism (Mas-Colell et al. 2005, page 878). It should be noted that while the VCG mechanism results in truthful implementation in dominant strategies, it does not guarantee budget balance. If the budget balance condition for all environments $e \in \mathcal{E}$ is required to be satisfied and $\mathcal{E}$ is sufficiently rich, then there is no social choice rule $\gamma: \mathcal{E} \rightarrow \mathcal{A}, \gamma(e):=$ $\left(x_{1}(e), x_{2}(e), \cdots, x_{N}(e)\right), t_{1}(e), t_{2}(e), \cdots, t_{N}(e)$ that is truthfully implementable in dominant strategies (Green \& Laffont 1979).

\subsubsection{Implementation in Nash equilibria}

In his seminal paper, Maskin (Maskin 1999) characterized social choice rules that can be implemented in NE. A key property that a social choice rule must possess in order to be implemented in NE is monotonicity (Maskin 1999). Monotonicity 
is satisfied by many social choice rules including the "social welfare maximizing correspondence" and the "Pareto correspondence" (Mas-Colell et al. 2005, Maskin n.d., Jackson 2001, Hurwicz, Maskin \& Postlewaite 1995). Monotonicity along with no veto power, (Maskin 1999), and $N \geq 3$, are sufficient conditions to guarantee implemtation of a social choice rule in NE. Weak no veto power is satisfied by most social choice rules (see example in (Maskin n.d.)).

Maskin's fundamental result is the following.

THEOREM 2 ((Maskin 1999)) If a social choice rule $\gamma: \mathcal{E} \rightarrow \mathcal{A}$ is implementable in NE then it must be monotonic. Furthermore, if $\gamma$ is monotonic and satisfies the weak no-veto power condition and the number of users is at least 3 , then $\gamma$ is implementable in $N E$.

Maskin's proof of Theorem 2 is constructive. Given a social choice rule $\gamma$ that satisfies monotonicity and weak no veto power, Maskin constructs a game form that implements $\gamma$. Maskin's mechanism requires, in general, an infinite dimensional message space (see (Maskin 1999)). That is why the engineering (Sharma \& Teneketzis 2009, Kakhbod \& Teneketzis 2012b, Kakhbod \& Teneketzis 2012c, Kakhbod \& Teneketzis 2012a, Stoenescu \& Ledyard 2006) and some of the economic literature ((Reichelstein \& Reiter 1988), (Groves \& Ledyard 1987)) on implementation in NE has not followed Maskin's approach. The methodology and mechanisms adopted in this chapter for implementation in NE will be presented in Section 3.

\subsubsection{Implementation in BNE}

In the Bayesian framework each agent $i \in \mathcal{N}$ knows its own environment $e_{i}$ and has a pdf $p\left(\cdot \mid e_{i}\right)$ on the set $\mathcal{E}_{-i}:=\mathcal{E}_{1} \times \mathcal{E}_{2} \times \ldots \times \mathcal{E}_{i-1} \times \mathcal{E}_{i+1} \times \ldots \times \mathcal{E}_{N}$ of environments of all other agents $j \neq i$. While the functional forms $p\left(\cdot \mid e_{j}\right)$ are known to all agents and the mechanism designer for all $e_{j} \in \mathcal{E}_{j}$, for all $j \in \mathcal{N}$, only agent $i$ knows the true realization of $e_{i}$.

In this situation there are results that identify properties of social choice rules under which implementation in BNE is assured. The revelation principle then ensures that truthful implementation in $\mathrm{BNE}$ is possible.

The key properties that a social choice rule $\gamma: \mathcal{E} \mapsto \mathcal{A}$ must possess so that it can be implemented in BNE are Bayesian incentive compatibility and Bayesian monotonicity. We formally introduce Bayesian incentive compatibility, as it is critical for our analysis in Section 4, and we briefly discuss Bayesian monotonicity.

DEFinition 4 (Bayesian Incentive Compatibility) A social choice function $\gamma: \mathcal{E} \mapsto \mathcal{A}$ is Bayesian incentive compatible if and only if for every $i \in \mathcal{N}$,

$$
\int_{\mathcal{E}_{-i}} u_{i}\left(\gamma\left(e_{i}, e_{-i}\right)\right) p\left(e_{-i} \mid e_{i}\right) d e_{-i} \geq \int_{\mathcal{E}_{-i}} u_{i}\left(\gamma\left(e_{i}^{\prime}, e_{-i}\right)\right) p\left(e_{-i} \mid e_{i}\right) d e_{-i},
$$

1 In Section 3, the social choice rule/goal correspondence is the social welfare maximizing or the Pareto correspondence. 
The intuition behind this condition is the following. Consider the mechanism $(\mathcal{E}, \gamma)$. The left hand side of $(0.9)$ is agent $i$ 's expected payoff when the environment is $e=\left(e_{i}, e_{-i}\right)$ and every agent (including $\left.i\right)$ uses a truthful strategy. The right hand side of (0.9) is agent $i$ 's expected payoff when the environment is $e=\left(e_{i}, e_{-i}\right)$, every agent $j \neq i$ uses a truthful strategy and agent $i$ reports $e_{i}^{\prime} \neq e_{i}$. The inequality in (0.9) says that the social choice function $\gamma$ is Bayesian incentive compatible if and only if the truthful strategy/truth telling is an equilibrium of the game induced by $(\mathcal{E}, \gamma)$. The collection of inequality conditions (0.9) is called Bayesian incentive compatibility conditions. Bayesian incentive compatibility is essential for implementation in BNE. Any social choice function that is not Bayesian incentive compatible cannot be implemented in BNE.

Bayesian monotonicity was defined and interpreted in (Palfrey \& Srivastava 1989, Palfrey \& Srivastava n.d.a, Jackson 1991). In (Palfrey \& Srivastava 1989), it is formally shown that Bayesian monotonicity is an extension of Maskin's monotonicity condition.

Bayesian incentive compatibility and Bayesian monotonicity are necessary conditions for the implementation of a social choice correspondence in BNE. Specifically the following result holds:

THEOREM 3 ((Palfrey \& Srivastava n.d.b), page 26) If the social choice correspondence $\gamma: \mathcal{E} \mapsto \mathcal{A}$ is implementable in BNE then,

$1 \gamma$ satisfies Bayesian monotonicity.

$2 \gamma$ is Bayesian incentive compatible.

Bayesian incentive compatibility, Bayesian monotonicity along with some additional technical conditions stated in (Jackson 1991, Palfrey \& Srivastava n.d.b, Palfrey \& Srivastava 1989, Palfrey 1994), are sufficient for the implementation of a social choice correspondence in BNE.

\section{Nash implementation for social welfare maximization and Pareto optimality}

In this section we consider power allocation and spectrum sharing problems arising in wireless networks where the goal correspondence is either the social welfare maximizing correspondence (Section 3.4) or the Pareto correspondence (Section 3.5). We formulate these problems within the framework of implementation theory that was presented in Section 2. We choose Nash equilibrium as a solution/equilibrium concept and we adopt Nash's "mass-action" interpretation of NE that we discuss in Section 3.6. We present mechanisms/game forms that are individually rational, budget balanced, and implement in Nash equilibria the social welfare correspondence or the Pareto correspondence, both defined in Section 3.2. 
Consider a wireless communication network consisting of spatially separated transmitters and receivers (some transmitters and receivers may be colocated as well). Each transmitter intends to communicate with a unique and fixed receiver; we call such a transmitter-receiver pair a user. Suppose there are $N$ users in the system. Let $\mathcal{N}:=\{1,2, \ldots, N\}$ denote the set of these users. Each user can communicate $^{2}$ over a set $\mathcal{B}:=\{1,2, \ldots, B\}$ of possible frequency bands. Within each frequency band $j \in \mathcal{B}$, a user $i \in \mathcal{N}$ can communicate using a transmission power ${ }^{3}$ level $p_{i j}$ which can lie either in a convex set $\mathcal{P}_{i j}:=\left[0, P_{i j}^{\max }\right]$ or in a discrete (quantized) set $\mathcal{Q}_{i j}:=\left\{\phi, Q_{i j}^{1}, Q_{i j}^{2}, \ldots, Q_{i j}^{\max }\right\}$. When the power levels are discrete, $p_{i j}=\phi$ implies that user $i$ does not use frequency band $j$. The total transmission power user $i \in \mathcal{N}$ can use across all frequency bands must lie within its transmission power capacity $P_{i}^{\text {total }}$, i.e. $\sum_{j \in \mathcal{B}} p_{i j} \leq P_{i}^{\text {total }}$.

If two or more users use the same frequency band to communicate and they are spatially close, they create interference to one another. The communication of users in a given frequency band $j \in \mathcal{B}$ and the resulting interference can be represented by a directed graph $\mathcal{G}_{j}$ as shown in Fig. 0.1. Each node in this graph represents a user. A directed edge from user $k$ to user $i$ indicates that the receiver of user $i$ is within reach of the transmitter of user $k$; hence if user $k$ uses a positive transmission power, it creates interference to user $i$ 's reception in the given frequency band. Based on the interference pattern, we can define a set $\mathcal{R}_{i j} \subset \mathcal{N}$ of user $i$ 's neighbors who can potentially create interference to its reception in frequency band $j$. Similarly, we can define a set $\mathcal{C}_{k j} \subset \mathcal{N}$ of user $k$ 's neighbors who would experience interference from its transmission in frequency band $j$. From the graph shown in Fig. 0.1, the neighbor sets $\mathcal{R}_{i j}$ and $\mathcal{C}_{k j}$ can be defined as follows:

$$
\begin{aligned}
& \mathcal{R}_{i j}:=\left\{k \in \mathcal{N} \mid k \rightarrow i \text { in } \mathcal{G}_{j}\right\}, \\
& \mathcal{C}_{k j}:=\left\{i \in \mathcal{N} \mid k \rightarrow i \text { in } \mathcal{G}_{j}\right\},
\end{aligned}
$$

i.e. $\mathcal{R}_{i j}$ is the set of users from whom there is a directed edge to user $i$, and $\mathcal{C}_{k j}$ is the set of users to which there is a directed edge from user $k$. Because of interference, the quality of a user's communication depends not only on the power of the signal received from its own transmitter but also on the powers of interfering signals. Let $h_{k i j}$ denote the channel gain between the transmitter of user $k$ and the receiver of user $i$ in frequency band $j$. This implies that in frequency band $j$, the signal transmitted by user $k$ with power $p_{k j}$ reaches user $i$ with power $\hat{p}_{k i j}:=$ $p_{k j} h_{k i j}$. Based on the received powers, the quality of a user's communication can be quantified by a valuation function $\hat{v}_{i}\left(\left(\hat{p}_{k i j}\right)_{k \in \mathcal{R}_{i j}, j \in \mathcal{B}}\right)$, where $\left(\hat{p}_{k i j}\right)_{k \in \mathcal{R}_{i j}, j \in \mathcal{B}}$ denotes the vector of powers of all signals received by user $i$ across all frequency

2 By communication of a user, we imply the communication between its corresponding transmitter and receiver.

3 The transmission power of a user implies the transmission power used by the corresponding transmitter. Similarly, whenever we say the power received by a user, it will imply the power received by the corresponding receiver. 
bands. If the channel gains $h_{k i j}, k \in \mathcal{R}_{i j}, j \in \mathcal{B}$, are known, then one can define

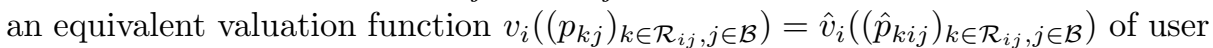
$i$ in terms of the transmission powers of the interference generating users. Thus the domain of $v_{i}$ is $\mathcal{D}_{i}:=\left\{\left(p_{k j}\right)_{k \in \mathcal{R}_{i j}, j \in \mathcal{B}} \mid p_{i j} \in \mathcal{P}_{i j}\left(\right.\right.$ or $\left.\mathcal{Q}_{i j}\right), j \in \mathcal{B} ; \sum_{j \in \mathcal{B}} p_{i j} \leq$ $P_{i}^{\text {total }} ; p_{k j} \in \mathbb{R}_{+}$(or $\mathcal{Q}_{k j}$ ), $\left.k \in \mathcal{R}_{i j} \backslash\{i\}, j \in \mathcal{B}\right\}$. Henceforth, in this chapter we will use the function $v_{i}(\cdot)$ to represent user $i$ 's valuation of the power and spectrum allocation for simplicity of exposition.

Now suppose that all users in the network are selfish and want to maximize their respective values from power and spectrum allocation. Suppose further that the users are strategic and hence may not want to reveal their true valuations of power and spectrum allocation. In order to provide incentives to these strategic users to reveal information that helps obtain social welfare maximizing allocations, each user $i \in \mathcal{N}$ is charged a tax $t_{i} \in \mathbb{R}$. This tax is set based on the users' messages according to a prespecified mechanism. As discussed in Section 2 it is desirable to achieve budget balance (i.e. $\sum_{i \in \mathcal{N}} t_{i}=0$ ) when taxes are involved. Budget balance requires collection and redistribution of taxes, and this is done by a network operator who is a separate entity from all the users, and who is not a profit maker.

Because a user's allocation preference is altered by the tax, to describe the overall satisfaction of a user from the value it receives from power and spectrum allocation and the tax it pays for it, we define a utility function $u_{i}\left(\left(p_{k j}\right)_{k \in \mathcal{R}_{i j}, j \in \mathcal{B}}, t_{i}\right)$ for each $i \in \mathcal{N}$ as follows:

$$
\begin{aligned}
& u_{i}\left(\left(p_{k j}\right)_{k \in \mathcal{R}_{i j}, j \in \mathcal{B}}, t_{i}\right):= \\
& \left\{\begin{array}{cc}
v_{i}\left(\left(p_{k j}\right)_{k \in \mathcal{R}_{i j}, j \in \mathcal{B}}\right)-t_{i}, & \text { if } p_{i j} \in \mathcal{P}_{i j}\left(\text { or } \mathcal{Q}_{i j}\right), j \in \mathcal{B} ; \sum_{j \in \mathcal{B}} p_{i j} \leq P_{i}^{\text {total }} ; \\
-\infty, & p_{k j} \in \mathbb{R}_{+}\left(\text {or } \mathcal{Q}_{k j}\right), k \in \mathcal{R}_{i j} \backslash\{i\}, j \in \mathcal{B},
\end{array}\right. \\
& -\infty \text { therwise. }
\end{aligned}
$$

We make the following assumptions on the wireless network model described above.

ASSUMPTION 1 For all $i \in \mathcal{N}$ and $j \in \mathcal{B}, \mathcal{R}_{i j}=\mathcal{C}_{i j}$, i.e. if user $i$ creates interference to some user $k$ in frequency band $j$, then user $k$ also creates interference to user $i$ in frequency band $j$.

Such a symmetry holds if the physical path of transmission from user $i$ to $j$ is similar to that from user $j$ to $i$.

Assumption 2 For each $i \in \mathcal{N}$ and $j \in \mathcal{B}$, the sets $\mathcal{R}_{i j}$ and $\mathcal{C}_{i j}$ are independent of the power allocation $p_{i j}, i \in \mathcal{N}, j \in \mathcal{B}$. In other words, these sets are solely determined by the spatial separation of the users; hence any change in transmission power levels does not change these sets as long as the spatial separation of users does not change over the time period of interest.

The above assumption holds in scenarios where the transmission power fluctuations resulting from a power allocation mechanism are not large. As a result, if 
two users are in the interference range of each other at some transmission power level, they continue to be in each other's interference range at any other power levels determined by the mechanism.

ASsumption 3 The network is static, i.e. the sets $\mathcal{N}, \mathcal{B}, \mathcal{P}_{i j}\left(\right.$ or $\left.\mathcal{Q}_{i j}\right), \mathcal{R}_{i j}, \mathcal{C}_{i j}$, $j \in \mathcal{B}, i \in \mathcal{N}$, the values $P_{i}^{\text {total }}, h_{k i j}, k \in \mathcal{R}_{i j}, j \in \mathcal{B}, i \in \mathcal{N}$, and the functions $v_{i}(\cdot), i \in \mathcal{N}$, do not change over the time period of interest.

The sets $\mathcal{P}_{i j}$ (or $\mathcal{Q}_{i j}$ ), $j \in \mathcal{B}, i \in \mathcal{N}$, and the transmission power capacities $P_{i}^{\text {total }}, i \in \mathcal{N}$, are constrained by the transmitter device capabilities and hence are static. On the other hand constant channel gains can be thought of as approximations of slowly changing cahnnel gains in networks where users have slow or zero mobility and the wireless channel conditions vary slowly compared to the time period of interest. The above factors together with Assumption 1 also imply that the set of users who create interference to one another continue to create the interference for the time period of interest; as a result the sets $\mathcal{R}_{i j}, \mathcal{C}_{i j}, j \in \mathcal{B}, i \in \mathcal{N}$, remain fixed.

ASSUMPtion 4 For each user $i \in \mathcal{N}$, its transmission capacity $P_{i}^{\text {total }}$, the sets $\mathcal{P}_{i j}$ (or $\mathcal{Q}_{i j}$ ), $j \in \mathcal{B}$, representing its transmission capability, and its valuation function $v_{i}(\cdot)$ are its private information. Each user $i \in \mathcal{N}$ also knows its neighbor sets $\mathcal{R}_{i j}, \mathcal{C}_{i j}, j \in \mathcal{B}$, and all channel gains $h_{k i j}, k \in \mathcal{R}_{i j}, j \in \mathcal{B}$.

The private knowledge of $P_{i}^{\text {total }}$ and the sets $\mathcal{P}_{i j}$ (or $\mathcal{Q}_{i j}$ ), $j \in \mathcal{B}$, is a result of the differences in transmitter devices of the users whose specifications may not be known to other users in the network. A detailed discussion of how each user can determine its neighbor sets and the respective channel gains is given in (Kakhbod \& Teneketzis 2012c).

Assumption 5 When $p_{i j} \in \mathcal{P}_{i j} \forall i \in \mathcal{N}, j \in \mathcal{B}$, the valuation function $v_{i}(\cdot)$ : $\mathcal{D}_{i} \rightarrow \mathbb{R}_{+}$is concave in its argument for each $i \in \mathcal{N}$.

It is shown in (Sharma \& Teneketzis 2009, Sharma \& Teneketzis 2011) that in a wireless network where users' use Code Division Multiple Access technology (CDMA) to transmit data and employ Minimum Mean Square Error Multi-User Detector (MMSE-MUD) receivers, the utility functions of the users are close to concave. Thus, the above assumption can be thought of as an approximation to such scenarios.

ASSUMPTION 6 Each user is selfish, strategic, and a self utility maximizer, i.e. each user $i \in \mathcal{N}$ intends to maximize its own utility $u_{i}\left(\left(p_{k j}\right)_{k \in \mathcal{R}_{i j}, j \in \mathcal{B}}, t_{i}\right)$.

The above scenario can arise very often in modern wireless networks where users possess privately operated devices that can be programed to optimize their respective utilities.

ASSUMPTION 7 The network operator does not derive any utility from power and spectrum allocation or tax distribution. It simply acts as an accountant collecting and redistributing the tax according to the specifications of the mechanism. 
In order to facilitate the collection and redistribution of tax, we also make the following assumption.

ASSUMPTION 8 The network operator knows all the sets $\mathcal{R}_{i j}, \mathcal{C}_{i j}, \forall j \in$ $\mathcal{B}, \forall i \in \mathcal{N}$ in the network, and all channel gains $h_{k i j}, \forall k \in \mathcal{R}_{i j}, \forall j \in$ $\mathcal{B}, \forall i \in \mathcal{N}$.

The process by which the network operator can determine the neighbor sets of all users and all the channel gains can be found in (Kakhbod \& Teneketzis $2012 c)$. The process used by the network operator also enables the users to determine their respective neighbor sets and channel gains as mentioned after Assumption 4.

Finally we make the following assumption about the common knowledge in the network.

ASSUMPtion 9 The set $\mathcal{B}$ of frequency bands and the facts given by Assumptions 1-8 are common knowledge among all the users and the network operator.

With the above assumptions, we formulate a power and spectrum allocation problem for the network $\left(M_{P S A}\right)$.

\section{The power allocation and spectrum sharing problem}

For the Model $\left(M_{P S A}\right)$ we wish to determine, under the decentralized information constraints implied by Assumptions 1-9, power and spectrum allocations that maximize the social welfare. Below we formulate this decentralized power and spectrum allocation problem in the framework of implementation theory introduced in Section 2. We first specify the environment space and the outcome space of the problem based on the description of Model $\left(M_{P S A}\right)$ in Section 3.1. We then define the goal correspondence, and specify the objective for the solution of the decentralized power and spectrum allocation problem.

- Environment space: The environment $\boldsymbol{e}_{\boldsymbol{i}}$ of each user $i \in \mathcal{N}$ consists of the sets $\mathcal{P}_{i j}$ (or $\left.\mathcal{Q}_{i j}\right), \mathcal{R}_{i j}, \mathcal{C}_{i j}, j \in \mathcal{B}$, the values $P_{i}^{\text {total }}, h_{k i j}, k \in \mathcal{R}_{i j}, j \in \mathcal{B}$, and the function $v_{i}(\cdot)$. The environment space $\mathcal{E}_{i}$ of user $i$ is the space comprising the following: the space of all convex sets $\mathcal{P}_{i j}=\left[0, P_{i j}^{\max }\right], P_{i j}^{\max } \in \mathbb{R}_{+}$, (or the space of all discrete sets $\mathcal{Q}_{i j}=\left\{\phi, Q_{i j}^{1}, Q_{i j}^{2}, \ldots, Q_{i j}^{\max }\right\}, Q_{i j}^{z} \in \mathbb{R}_{+}, z \in$ $\{1,2, \ldots, \max \})$, the space of all subsets $\mathcal{R}_{i j}, \mathcal{C}_{i j}, j \in \mathcal{B}$, of $\mathcal{N}$, the space of all non-negative real values $P_{i}^{\text {total }}$, the space of all non-negative real valued channel gains $h_{k i j}, k \in \mathcal{R}_{i j}, j \in \mathcal{B}$, and the space of all concave functions $v_{i}$.

- Outcome space: The outcome space $\mathcal{A}$ is the set

$$
\mathcal{D}:=\left\{(\boldsymbol{p}, \boldsymbol{t}) \mid p_{i j} \in \mathcal{P}_{i j}\left(\text { or } \mathcal{Q}_{i j}\right), \sum_{j \in \mathcal{B}} p_{i j} \leq P_{i}^{\text {total }}, i \in \mathcal{N}, j \in \mathcal{B} ; \sum_{i \in \mathcal{N}} t_{i}=0\right\} .
$$

We call all allocations $(\boldsymbol{p}, \boldsymbol{t}) \in \mathcal{D}$ feasible allocations. 
- Goal correspondence: For the class of problems discussed in Section 3.4 the goal correspondence $\gamma$ is the social welfare correspondence. It is defined as the mapping from $\mathcal{E}=\otimes_{i \in \mathcal{N}} \mathcal{E}_{i}$ to $\mathcal{D}$ which maps each environment $e \in \mathcal{E}$ (i.e. each instance of users' utility functions $u_{i} \in \boldsymbol{e}_{\boldsymbol{i}}, i \in \mathcal{N}$ ) to the set of optimal solutions of the following centralized allocation problem:

Problem $\left(P_{P S A}^{C}\right)$

$$
\begin{aligned}
\max _{(\boldsymbol{p}, \boldsymbol{t}) \in\left(\mathbb{R}^{N B} \times \mathbb{R}^{N}\right)} & \sum_{i \in \mathcal{N}} u_{i}\left(\left(p_{k j}\right)_{k \in \mathcal{R}_{i j}, j \in \mathcal{B}}, t_{i}\right) \\
\text { s.t. } & \sum_{i \in \mathcal{N}} t_{i}=0
\end{aligned}
$$

For the problem discussed in Section 3.5 the goal correspondence $\gamma: \mathcal{E} \rightarrow \mathcal{D}$ is the Pareto correspondence that is defined as follows. For every $\boldsymbol{e} \in \mathcal{E}$, each $(\boldsymbol{p}, \boldsymbol{t}) \in \gamma(\boldsymbol{e})$ has the following property. There is no $\left(\boldsymbol{p}^{\prime}, \boldsymbol{t}^{\prime}\right) \in \mathcal{D}$ such that $u_{j}\left(\boldsymbol{p}^{\prime}, \boldsymbol{t}^{\prime}\right) \geq u_{j}(\boldsymbol{p}, \boldsymbol{t})$ for all $j \in \mathcal{N}$, and $u_{i}\left(\boldsymbol{p}^{\prime}, \boldsymbol{t}^{\prime}\right)>u_{i}(\boldsymbol{p}, \boldsymbol{t})$ for some $i \in \mathcal{N}$ $\left(u_{i} \in \boldsymbol{e}_{\boldsymbol{i}}\right.$ for all $\left.i \in \mathcal{N}\right)$.

- Objective: We wish to develop game forms $(\mathcal{M}, h)$ that:

- implement in Nash equilibria the social welfare correspondence (Section 3.4) or the Pareto correspondence (Section 3.5) defined above;

- are individually rational;

- are budget balanced.

In the next section we develop a conceptual framework that must guide the construction of game forms which possess the above properties. In subsequent sections we will investigate special cases of Model $M_{P S A}$; for these cases we will present specific game forms that are designed within the framework developed below.

3.3 Constructing a game form for the decentralized power and spectrum allocation problem

We present guidelines for the construction of a game form when the goal correspondence is the social welfare maximizing correspondence. Similar ideas apply to the design of game forms when the goal correspondence is the Pareto correspondence.

We begin with a discussion on the construction of the message space. Since a solution for Problem $\left(P_{P S A}^{C}\right)$ consists of the power and tax profiles of the users, the message exchange among the users should contain information that is helpful in determining the optimal values of these profiles. Note that each user's utility is affected by the power allocation of a subset of network users. Therefore, each user should have a contribution in determining the power allocations of those neighbors that affect its utility. Furthermore, a user should make a payment (positive or negative) for the power allocations of all such neighbors because they all impact its utility. Since each neighbor's allocation makes a different 
impact on the user's utility, the user may make different payments for each neighbor's allocation. One way to take into account the above two factors is to let each user communicate as its message/strategy a proposal that consists of two components: one that indicates what power allocations the user wants for its neighbors; and the other that indicates the price the user wants to pay for the power allocations of each of its neighbors.

We next discuss the construction of the outcome function. The specification of the outcome function is arguably the most important and challenging task in the construction of a game form/decentralized resource allocation mechanism. Because the designer of the mechanism cannot alter the users' utility functions $u_{i}, i \in \mathcal{N}$, the only way it can achieve the objectives of Nash implementation, budget balance, and individual rationality is through the provision of appropriate tax functions/incentives that induce strategic users to follow the mechanism's operational rules. Below we develop the guidelines for the construction of outcome functions that achieve each of the above objectives.

To achieve implementation in NE, the outcome function must make sure that all NE of the message exchange (that is done according to the discussion presented above) lead to optimal centralized allocations. This suggests that the outcome function must induce price taking behavior ${ }^{4}$ for all users at all NE. If price taking behavior is induced, then, through NE price control, the mechanism can induce the users to take actions that are optimal for their own objective as well as for the centralized problem $\left(P_{P S A}^{C}\right)$. As discussed in the previous paragraph, a user should make a payment for the power allocations of each of its neighbors that affect its utility. In order for the mechanism to induce price taking behavior, the NE price that a user $i \in \mathcal{N}$ pays for its neighbors' allocations must depend only on the messages/proposals of users other than $i$. Thus, the NE tax of user $i, i \in \mathcal{N}$, must be of the form $\sum_{j \in \mathcal{B}} \sum_{k \in \mathcal{R}_{i j}} l_{i k j}^{*} p_{k j}^{*}$ where $p_{k j}^{*}$ is the NE power allocation of user $k$ in frequency band $j$ and $l_{i k j}^{*}$ is the NE price of this allocation for user $i$ that is independent of user $i$ 's message. With the NE tax form $\sum_{j \in \mathcal{B}} \sum_{k \in \mathcal{R}_{i j}} l_{i k j}^{*} p_{k j}^{*}$, each user $i \in \mathcal{N}$ can influence its NE utility only through the allocations $p_{k j}^{*}, k \in \mathcal{R}_{i j}, j \in \mathcal{B}$. Since each user's utility is its private information, the utility maximizing allocations of a user are known only to that user. Therefore, to allow each user to obtain its utility maximizing allocations at given NE prices, the outcome function must provide each user $i \in \mathcal{N}$ an independent control, through its power profile proposal, over each of the allocations $p_{k j}^{*}, k \in \mathcal{R}_{i j}, j \in \mathcal{B}$. In other words, each allocation $p_{k j}^{*}, j \in \mathcal{B}$, must be independently controlled by each of the users $i \in \mathcal{C}_{k j}, j \in \mathcal{B}$, and this fact should be reflected in the form of the outcome function.

4 An individual/user which is not influential enough to affect the equilibrium prices reached by a mechanism is called price taker which we consider it as a non-strategic user. In other words, price taking behavior that leads to a solution of the centralized problem, means that individuals/users act as if their behavior has no effect on the equilibrium prices where as, inducing price taking behavior means that users who are non-price taking (strategic) behave as if they are price taker. 
To achieve budget balance, the NE prices $l_{i j}^{*}, j \in \mathcal{R}_{i}, i \in \mathcal{N}$, must satisfy

$$
\sum_{i \in \mathcal{N}} \sum_{j \in \mathcal{B}} \sum_{k \in \mathcal{R}_{i j}} l_{i k j}^{*} p_{k j}^{*}=0
$$

or, equivalently, ${ }^{5}$

$$
\sum_{j \in \mathcal{B}} \sum_{k \in \mathcal{N}} \sum_{i \in \mathcal{C}_{k j}} l_{i k j}^{*} p_{k j}^{*}=0 .
$$

One way to satisfy the requirement in (0.13) is to set for each $j \in \mathcal{B}$ and $k \in \mathcal{N}$, $\sum_{i \in \mathcal{C}_{k j}} l_{i k j}^{*}=0$.

The features of the outcome function discussed so far could lead to price taking behavior and budget balance. However, the construction of an outcome function which has only the above features may lead to the following difficulty. Because each user knows that its price proposal does not affect its own tax and hence, its utility, it may propose arbitrary prices for its neighbors in its price proposal. One way to overcome this difficulty without altering price taking behavior and budget balance is to add a "penalty" term in the tax function of each user. To preserve the price taking behavior of the users at NE, the penalty should be imposed only at off NE messages. This penalty should depend on each user's own price proposal and it should increase with the user's price proposal. However, to avoid unnecessary penalties, the penalty of a user should be reduced if its power profile proposal for its neighbors is in agreement with other users' proposals.

Adding to the tax function a penalty term with the above characteristics may result in an unbalanced budget. To preserve budget balance a "budget balancing" term should be added to the tax function of each user. This term must balance the net flow of the money due to the penalty term. Because the penalty is imposed on the users only at off NE messages, the budget balancing term must influence the users' tax only at off NE messages. To prevent the budget balancing term from altering a user's strategic behavior that is governed by the price taking term and the penalty term in the user's tax, the budget balancing term should be independent of the user's own message.

To achieve individual rationality the outcome function must make sure that at all NE, the utility of each user is at least as much as the utility this user gets by not participating in the allocation process (cf. Section 2.1) . This property is automatically achieved if the outcome function has the following features discussed earlier: (i) It induces price taking behavior; and (ii) It gives each user an independent control over in determining the power/spectrum allocations that affect its utility. If each user can control the allocations that affect its NE utility, for any set of NE prices $l_{i k j}^{*}, k \in \mathcal{R}_{i j}, j \in \mathcal{B}$, a user $i \in \mathcal{N}$ can force all the allocations $p_{k j}^{*}, k \in \mathcal{R}_{i j}, j \in \mathcal{B}$, to be 0 , thereby also making its NE payment $\sum_{j \in \mathcal{B}} \sum_{k \in \mathcal{R}_{i j}} l_{i k j}^{*} p_{k j}^{*}=0$. Thus, with the above features of the outcome func-

${ }^{5}$ From the construction of the graph matrix $\mathcal{G}_{j}$ and the sets $\mathcal{R}_{i j}$ and $\mathcal{C}_{k j}, i, k \in \mathcal{N}$, the sum $\sum_{i \in \mathcal{N}} \sum_{k \in \mathcal{R}_{i j}}(\cdot)$ is equivalent to the sum $\sum_{k \in \mathcal{N}} \sum_{i \in \mathcal{C}_{k j}}(\cdot), \quad \forall j \in \mathcal{B}$. 
tion, each user can independently guarantee a minimum of zero utility for itself which is precisely the utility it gets by not participating in the allocation process.

\section{Social welfare maximizing power allocation in a single frequency band}

In this section we consider a special case of Model $\left(M_{P S A}\right)$ where $B=1$, i.e. users communicate in a single frequency band, and $p_{i j} \in \mathcal{P}_{i j}, \forall i \in \mathcal{N}, j=1$, i.e. the users can select their transmission power levels from a convex set. The goal correspondence $\gamma$ is the social welfare maximizing correspondence, defined in Section 3.2, which is implementable in NE (cf. Section 2.4.2, (Mas-Colell et al. 2005), (Maskin n.d., Jackson 2001, Hurwicz et al. 1995)). Because there is only one frequency band, we don't consider the additional constraint $p_{i 1} \leq$ $P_{i}^{\text {total }}$. To simplify the discussion in this section we drop the index $j$ from our notation and write $p_{i j}, h_{k i j}, \mathcal{P}_{i j}, \mathcal{R}_{i j}$ and $\mathcal{C}_{i j}$ as $p_{i}, h_{k i}, \mathcal{P}_{i}, \mathcal{R}_{i}$ and $\mathcal{C}_{i}$ respectively. We call this special case of Model $\left(M_{P S A}\right)$ as Model $\left(M_{P S A}^{1}\right)$. The model $\left(M_{P S A}^{1}\right)$ includes in it as a special case (corresponding to $\mathcal{R}_{i}=\mathcal{C}_{i}=\mathcal{N}$ ) the public goods model studied in (Hurwicz 1979); thus the design of power allocation mechanism for Model $\left(M_{P S A}^{1}\right)$ draws inspiration from the public goods allocation mechanism presented in (Hurwicz 1979).

Below we present a game form $(\mathcal{M}, h)$ for Model $\left(M_{P S A}^{1}\right)$ that is inspired by the game form of (Hurwicz 1979) and is developed according to the guidelines discussed in Section 3.3.

The message space:

For each user $i \in \mathcal{N}$, its message space $\mathcal{M}_{i}=\mathbb{R}^{\left|\mathcal{R}_{i}\right|} \times \mathbb{R}_{+}^{\left|\mathcal{R}_{i}\right|}$, where $\left|\mathcal{R}_{i}\right|$ denotes the cardinality of set $\mathcal{R}_{i}$. A message $\boldsymbol{m}_{i}$ in this message space can be written as:

$$
\begin{aligned}
\boldsymbol{m}_{\boldsymbol{i}} & =\left({ }^{\boldsymbol{i}} \varrho_{\mathcal{R}_{\boldsymbol{i}}},{ }^{\boldsymbol{i}} \boldsymbol{\pi}_{\mathcal{R}_{\boldsymbol{i}}}\right), \quad{ }^{\boldsymbol{i}} \varrho_{\mathcal{R}_{\boldsymbol{i}}} \in \mathbb{R}^{\left|\mathcal{R}_{i}\right|},{ }^{\boldsymbol{i}} \boldsymbol{\pi}_{\mathcal{R}_{\boldsymbol{i}}} \in \mathbb{R}_{+}^{\left|\mathcal{R}_{i}\right|}, \\
\text { where, } \quad{ }^{\boldsymbol{i}} \varrho_{\mathcal{R}_{\boldsymbol{i}}} & :=\left({ }^{i} \varrho_{k}\right)_{k \in \mathcal{R}_{i}}, \quad{ }^{\boldsymbol{i}} \boldsymbol{\pi}_{\mathcal{R}_{\boldsymbol{i}}}:=\left({ }^{i} \pi_{k}\right){ }_{k \in \mathcal{R}_{i}} .
\end{aligned}
$$

In the above message, ${ }^{i} \varrho_{k}$ can be thought of as the transmission power proposal for user $k, k \in \mathcal{R}_{i}$, by user $i, i \in \mathcal{N}$. Similarly, ${ }^{i} \pi_{k}$ can be thought of as the price that user $i, i \in \mathcal{N}$, proposes to pay for the transmission power of user $k, k \in \mathcal{R}_{i}$. Note that the above message space follows the structure suggested in Section 3.3.

Using the above message space, each user $i \in \mathcal{N}$ sends its message $\boldsymbol{m}_{\boldsymbol{i}}$ to the network operator, and the component $\left({ }^{i} \varrho_{k},{ }^{i} \pi_{k}\right)$ of its message to its neighbor $k \in \mathcal{R}_{i}$. After the users communicate their messages to the network operator, it computes their transmission powers and taxes according to the following outcome function.

\section{The outcome function:}

The outcome function $h$ has the form $h(\boldsymbol{m})=\left(p_{i}\left(\boldsymbol{m}_{\mathcal{C}_{\boldsymbol{i}}}\right), t_{i}\left(\left(\boldsymbol{m}_{\mathcal{C}_{\boldsymbol{k}}}\right)_{k \in \mathcal{R}_{i}}\right)\right)_{i \in \mathcal{N}}$, i.e. it consists of $2 N$ functions - one for determining the transmission power, and one for determining the tax for each user $i \in \mathcal{N}$. The transmission power of each 
user is determined as follows.

$$
p_{i}\left(\boldsymbol{m}_{\mathcal{C}_{i}}\right)=\frac{1}{\left|\mathcal{C}_{i}\right|} \sum_{k \in \mathcal{C}_{i}}{ }^{k} \varrho_{i}, \quad i \in \mathcal{N} .
$$

To determine the users' taxes the network operator considers each set $\mathcal{C}_{k}, k \in \mathcal{N}$, and assigns indices $1,2, \ldots,\left|\mathcal{C}_{k}\right|$ in a cyclic order to the users in $\mathcal{C}_{k}$. Each index $1,2, \ldots,\left|\mathcal{C}_{k}\right|$ is assigned to an arbitrary but unique user $i \in \mathcal{C}_{k}$. Once the indices are assigned to the users in each set $\mathcal{C}_{k}$, they remain fixed throughout the time period of interest. We denote the index of user $i$ associated with set $\mathcal{C}_{k}$ by $I_{i k}$. The index $I_{i k} \in\left\{1,2, \ldots,\left|\mathcal{C}_{k}\right|\right\}$ if $i \in \mathcal{C}_{k}$, and $I_{i k}=0$ if $i \notin \mathcal{C}_{k}$. Since for each set $\mathcal{C}_{k}$, each index $1,2, \ldots,\left|\mathcal{C}_{k}\right|$ is assigned to a unique user $i \in \mathcal{C}_{k}$, for all $i, r \in \mathcal{C}_{k}$ such that $i \neq r, I_{i k} \neq I_{r k}$. Note also that for any user $i \in \mathcal{N}$, and any $k, r \in \mathcal{R}_{i}$, the indices $I_{i k}$ and $I_{i r}$ are not necessarily the same and are independent of each other. We denote the user with index $x \in\left\{1,2, \ldots,\left|\mathcal{C}_{k}\right|\right\}$ in set $\mathcal{C}_{k}$ by $\mathcal{C}_{k(x)}$. Thus, $\mathcal{C}_{k\left(I_{i k}\right)}=i$ for $i \in \mathcal{C}_{k}$. The cyclic order indexing means that, if $I_{i k}=\left|\mathcal{C}_{k}\right|$, then $\mathcal{C}_{k\left(I_{i k}+1\right)}=\mathcal{C}_{k(1)}, \mathcal{C}_{k\left(I_{i k}+2\right)}=\mathcal{C}_{k(2)}$, and so on. In Fig. 0.2 we illustrate the above indexing rule for the set $\mathcal{C}_{k}$ shown in Fig. 0.1.

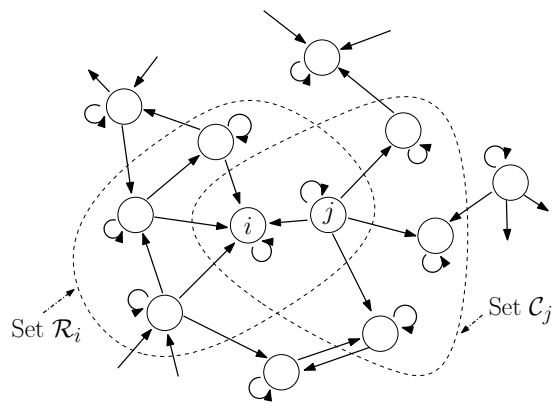

Figure 0.1 A local public good network depicting the Neighbor sets $\mathcal{R}_{i}$ and $\mathcal{C}_{j}$ of users $i$ and $j$ respectively.

Based on the above indexing, the users' taxes $t_{i}, i \in \mathcal{N}$, are determined as follows.

$$
\begin{aligned}
t_{i}\left(\left(\boldsymbol{m}_{\mathcal{C}_{k}}\right)_{k \in \mathcal{R}_{i}}\right)= & \sum_{k \in \mathcal{R}_{i}} l_{i k}\left(\boldsymbol{m}_{\mathcal{C}_{k}}\right) p_{k}\left(\boldsymbol{m}_{\mathcal{C}_{k}}\right)+\sum_{k \in \mathcal{R}_{i}}{ }^{i} \pi_{k}\left({ }^{i} \varrho_{k}-{ }^{\mathcal{C}_{k\left(I_{i k}+1\right)}} \varrho_{k}\right)^{2} \\
& -\sum_{k \in \mathcal{R}_{i}} \mathcal{C}_{k\left(I_{i k}+1\right)} \pi_{k}\left({ }^{\mathcal{C}_{k\left(I_{i k}+1\right)}} \varrho_{k}-{ }^{\mathcal{C}_{k\left(I_{i k}+2\right)}} \varrho_{k}\right)^{2}, \quad i \in \mathcal{N}
\end{aligned}
$$

$$
\text { where, } \quad l_{i k}\left(\boldsymbol{m}_{\mathcal{C}_{\boldsymbol{k}}}\right)={ }^{\mathcal{C}_{k\left(I_{i k}+1\right)}} \pi_{k}-{ }^{\mathcal{C}_{k\left(I_{i k}+2\right)}} \pi_{k}, \quad k \in \mathcal{R}_{i}, \quad i \in \mathcal{N} .
$$

Note that the construction of the tax function in (0.16) follows the guidelines presented in Section 3.3. Specifically, the tax of each user consists of three terms. The first term is the one that induces price taking behavior as it does not depend on the price proposal of user $i$. Note that the construction of $l_{i k}$ in (0.17) also satisfies the condition in (0.13). The second term in $(0.16)$ is the penalty term 


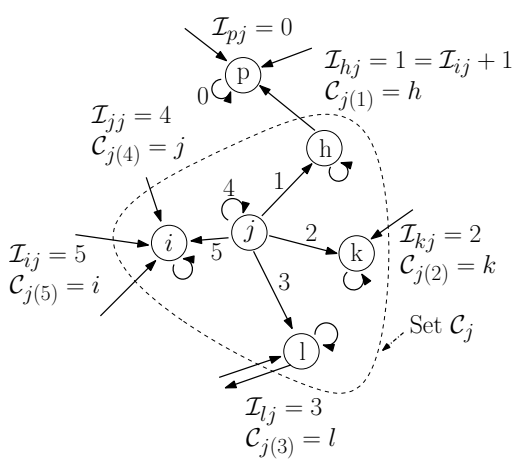

Figure 0.2 Illustration of indexing rule for set $\mathcal{C}_{j}$ shown in Fig. 0.1. Index $\mathcal{I}_{r j}$ of user $r \in \mathcal{C}_{j}$ is indicated on the arrow directed from $j$ to $r$. The notation to denote these indices and to denote the user with a particular index is shown outside the dashed boundary demarcating the $\operatorname{set} \mathcal{C}_{j}$.

which increases with each of the price proposals ${ }^{i} \pi_{k}, k \in \mathcal{R}_{i}$, of user $i$. Finally, the third term in (0.16) which is similar to the second term (except the change in the index from $I_{i k}$ to $\left.I_{i k}+1\right)$ is the budget balancing term which balances the monetary exchange due to the penalty term; this term does not depend on the message of user $i$, thus its inclusion in the tax $t_{i}(\cdot)$ does not alter user $i$ 's straetgic behavior.

\section{Properties of the game form}

The characteristics of the game form described above help achieve the objectives stated in Section 3.2 as established by the following theorem.

THEOREM 4 ((Sharma \& Teneketzis 2012, Sharma 2009)) The game form $(\mathcal{M}, h)$ presented in Section 3.4 possesses the following properties:

(i) It implements ${ }^{6}$ in Nash equilibria the social welfare correspondence $\gamma$ (defined in Section 3.2) for the Model $\left(M_{P S A}^{1}\right)$.

(ii) It is budget balanced at all NE and off equilibria, i.e. the sum of the users' taxes is zero at all message profiles.

(iii) It is individually rational, i.e. each user voluntarily participates in the allocation process and obtains a non-negative utility at all NE.

\section{Pareto optimal power and spectrum allocation}

In this section we consider power allocation in multiple frequency bands. The goal correspondence $\gamma: \mathcal{E} \rightarrow \mathcal{A}$ is the Pareto correspondence, defined in Sec-

6 The implementation is only with respect to the transmission power profile $\boldsymbol{p}$, i.e. every Nash equilibrium message profile leads to a power allocation $\boldsymbol{p}^{*}$ that forms an optimal solution of Problem $\left(P_{P S A}^{C}\right)$, and every power allocation $\boldsymbol{p}^{*}$ that forms an optimal solution of Problem $\left(P_{P S A}^{C}\right)$ can be obtained through some Nash equilibrium of the game induced by the game form $(\mathcal{M}, h)$. 
tion 3.2, that is implementable in NE (cf. Section 2.4.2, (Mas-Colell et al. 2005), (Maskin n.d., Jackson 2001, Hurwicz et al. 1995)). We consider a special case of Model $\left(M_{P S A}\right)$ where $\mathcal{R}_{i j}=\mathcal{C}_{i j}=\mathcal{N} \forall i \in \mathcal{N}, j \in \mathcal{B}$, i.e. if a user transmits with positive power in some frequency band, then it creates interference to all the users in that band. Furthermore, we consider the case where the transmission power level $p_{i j}, j \in \mathcal{B}, i \in \mathcal{N}$, lies in a discrete (quantized) set $\mathcal{Q}_{i j}:=\left\{\phi, Q_{i j}^{1}, Q_{i j}^{2}, \ldots, Q_{i j}^{\max }\right\}$. We call this special case of Model $\left(M_{P S A}\right)$ as $\left(M_{P S A}^{2}\right)$.

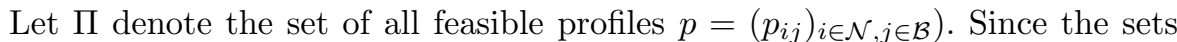
$\mathcal{N}, \mathcal{B}$ and $\mathcal{Q}$ are finite, $\Pi$ is finite. Let $|\Pi|=G_{N}$; we represent every feasible power profile by a number between 1 and $G_{N}$. Thus, $\Pi=\left\{1,2, \cdots, G_{N}\right\}$. Following the guidelines discussed in Section 3.3, we propose a game form the components of which are described as follows.

\section{The message space:}

The message space for user $i, i \in \mathcal{N}$, is given by $\mathcal{M}_{i} \subseteq \mathbb{Z} \times \mathbb{R}_{+}$, where $\mathbb{Z}$ and $\mathbb{R}_{+}$are the sets of integers and non-negative real numbers, respectively. Specifically, a message of user $i$ is of the form, $\mathbf{m}_{i}=\left(n_{i}, \pi_{i}\right)$ where $n_{i} \in \mathbb{Z}$ and $\pi_{i} \in \mathbb{R}_{+}$.

The meaning of the message space is the following. The component $n_{i}$ represents the power profile proposed by user $i$; the component $\pi_{i}$ denotes the price user $i$ is willing to pay per unit of the power profile $n_{i}$. The message $n_{i}$ belongs to an extended set $\mathbb{Z}$ of power profiles. Every element/integer in $\mathbb{Z}-\Pi$ corresponds to a power profile that is non-feasible.

\section{The outcome function:}

The outcome function $h$ is given by $h: \mathcal{M} \rightarrow \mathbb{N} \times \mathbb{R}^{N}$, and is defined as follows. For any $\mathbf{m}:=\left(\mathbf{m}_{1}, \mathbf{m}_{2}, \cdots, \mathbf{m}_{N}\right) \in \mathcal{M}$,

$$
h(\mathbf{m})=h\left(\mathbf{m}_{1}, \mathbf{m}_{2}, \cdots, \mathbf{m}_{N}\right)=\left(\widehat{I_{a v g}}(\boldsymbol{n}), t_{1}(\mathbf{m}), \cdots, t_{N}(\mathbf{m})\right) .
$$

where $I_{\text {avg }}(\mathbf{n})=I_{\text {avg }}\left(n_{1}, n_{2}, \cdots, n_{N}\right):=\left\lceil\frac{1}{N} \sum_{i=1}^{N} n_{i}\right\rceil$ (integer closest from above to the average $\left.\frac{1}{N} \sum_{i=1}^{N} n_{i}\right)$, and $\widehat{I_{\text {avg }}}(\boldsymbol{n})=I_{\text {avg }}(\mathbf{n}) 1\left\{I_{\text {avg }}(\boldsymbol{n}) \in \Pi\right\}$, where $1\{A\}$ denotes the indicator function of event $A$, that is, $1\{A\}=1$ if $A$ is true and $1\{A\}=0$, otherwise.

The component $t_{i}, i \in \mathcal{N}$, describes the tax (subsidy) that user $i$ pays (receives). The tax(subsidy) for every user is defined as follows,

$$
\begin{aligned}
t_{i}(\mathbf{m})= & \left\{I_{\text {avg }}(\mathbf{n})\left[\frac{\pi_{i+1}-\pi_{i+2}}{N}\right]+\left(n_{i}-n_{i+1}\right)^{2} \pi_{i}-\left(n_{i+1}-n_{i+2}\right)^{2} \pi_{i+1}\right\} \times \\
& 1\left\{I_{\text {avg }}(\mathbf{n}) \in \Pi\right\}
\end{aligned}
$$

where $N+1$ and $N+2$ are to be interpreted as 1 and 2, respectively.

\section{Interpretation of the Mechanism}


As pointed out in section 3.2, the design of an efficient resource allocation mechanism has to achieve the following goals. (i) It must induce strategic users to voluntarily participate in the allocation process. (ii) It must induce strategic users to follow its operational rules. (iii) It must result in weakly Pareto optimal allocations at all equilibria of the induced game. (iv) It must result in a balanced budget at all $\mathrm{NE}$ and off equilibrium.

To achieve these goals we propose the tax incentive function described by (0.18). This function consists of three components, $\Xi_{1}, \Xi_{2}$ and $\Xi_{3}$, that is,

$$
t_{i}(\mathbf{m})=\underbrace{I_{\text {avg }}(\boldsymbol{n})\left[\frac{\pi_{i+1}-\pi_{i+2}}{N}\right]}_{\Xi_{1}}+\underbrace{\left(n_{i}-n_{i+1}\right)^{2} \pi_{i}}_{\Xi_{2}} \underbrace{-\left(n_{i+1}-n_{i+2}\right)^{2} \pi_{i+1}}_{\Xi_{3}}(0
$$

The term $\Xi_{1}$ specifies the amount that each user must pay for the power profile which is determined by the mechanism. The price per unit of power, $\frac{\pi_{i+1}-\pi_{i+2}}{N}$, paid by user $i, i=1,2, \cdots, N$, is not controlled by that user. The terms $\Xi_{2}$ considered collectively provide an incentive to all users to propose the same power profile. The term $\Xi_{3}$ is not controlled by user $i$, its goal is to lead to a balanced budget.

\section{Properties of the game form}

The characteristics of the game form described above help achieve the objectives stated in Section 3.2 as established by the following theorem.

THEOREM 5 ((Kakhbod \& Teneketzis 2012c)) The game form presented in Section 3.5 possesses the following properties:

(i) It implements in NE the Pareto correspondence $\gamma$ (defined in Section 3.2) for the Model $\left(M_{P S A}^{2}\right)$.

(ii) It is budget balanced at all NE and off equilibria, i.e. the sum of the users' taxes is zero at all message profiles.

(iii) It is individually rational, i.e. each user voluntarily participates in the allocation process and obtains a non-negative utility at all NE.

\section{Interpreting Nash Equilibrium}

In the game theory literature, Nash equilibrium is a solution concept for games of complete information, that is, for games where the users' utilities are common knowledge among them. Nash equilibrium has been used as a solution concept in the power allocation and spectrum sharing problems we discussed in Sections 3.4 and 3.5, even though each user's utility is its own private information. We now discuss why NE is an appropriate solution concept for our problems.

In his original work (J. Nash 1950), Nash presented two interpretations of Nash equilibrium. The first is "mass-action" interpretation of NE points. According to this interpretation, it is unnecessary to assume that agents participating in the game have full knowledge of the structure of the game, or the ability to go 
through any complex reasoning process. But it is assumed that the participants have the ability to accumulate empirical information, obtained through repeated plays of the game and to evaluate, using this empirical information, the relative advantage of the various pure strategies they have at their disposal. The evaluation of empirical information determines, as the number of repeated plays of the game increases, the agents' NE strategies. Implicit in this interpretation of NE is the assumption that the game's environment $e$ is stable, that is, it does not change before the agents reach their equilibrium strategies. Within the context of mechanism design, Nash's "mass-action" interpretation of NE has also been adopted by several economists including Reichelstein and Reiter (Reichelstein \& Reiter 1988), and Groves and Ledyard (Groves \& Ledyard 1987). The authors of (Reichelstein \& Reiter 1988), (Groves \& Ledyard 1987) consider resource allocation problems with strategic agents who have private information, adopt NE as the solution concept and state: "We interpret our analysis as applying to an unspecified (message exchange) process in which users grope to a stationary message and in which the Nash property is a necessary condition for stationarity" (Reichelstein and Reiter page 664 (Reichelstein \& Reiter 1988)). "We do not suggest that each agent knows the environment when he computes his equilibrium strategy/message....We do suggest, however, that "complete information" Nash equilibrium messages may be the possible equilibrium of the iterative processthat is, stationary messages - just as the demand equal supply price is thought of as the equilibrium of some unspecified market dynamic process" (Groves and Ledyard, (Groves \& Ledyard 1987), pp. 69-70).

Nash's mass-action interpretation of NE has also been adopted in engineering publications where mechanism design ideas are used to solve decentralized resource allocation problems (see (Stoenescu \& Ledyard 2006, Sharma \& Teneketzis 2011, Kakhbod \& Teneketzis 2012b, Kakhbod \& Teneketzis 2012c, Kakhbod \& Teneketzis 2012a, Sharma \& Teneketzis 2012, Kash \& Parkes 2010)). Our interpretation of NE is similar to that of (Reichelstein \& Reiter 1988),(Groves $\&$ Ledyard 1987). The mechanisms proposed in Sections 3.4 and 3.5 are in equilibrium form like the mechanism in (Reichelstein \& Reiter 1988). Assumptions A2 and A3, appearing in Section 3.1, ensure that the environment of the problems discussed in Sections 3.4 and 3.5 is stable (as pointed out earlier, the stability of the problem's environment is an implicit requirement in the mass-action interpretation of $\mathrm{NE}$ ).

In the second interpretation of $\mathrm{NE}$, it is assumed that the agents know the full structure of the game in order to be able to predict the equilibrium strategies. This interpretation of NE is rationalistic and idealizing.

Other approaches to power allocation and spectrum sharing

In addition to the implementation theory approach presented in this section, there are other approaches to power allocation and spectrum sharing problems 
based either on other concepts of mechanism design or on game theory. We briefly describe these approaches below.

Alternative approaches based on mechanism design (e.g. auctions, VCG mechanisms) have been used to investigate spectrum and power allocation problems. It is well known that Federal Communications Commission (FCC) has used auctions to award spectrum since 1994 (Bajari \& Yeo 2009). Even at scales much smaller than that of FCC auctions, auctions have been studied for resource allocation in wireless networks. Examples of first-price and second-price sealed-bid auctions for spectrum allocation can be found in (Chen, Yu, Zhang, Chen \& Qiu 2008). These mechanisms are based on non monetary payments; in particular the price to pay for the spectrum opportunities is the spectrum sensing effort. In (Huang, Berry \& Honig 2004, Huang, Berry \& Honig 2006) VCG based auctions/mechanisms are presented for power and SINR (signal to interference and noise ratio) allocation subject to "an interference temperature constraint". These mechanisms maximize social welfare at truth-telling equilibria. In the mechanisms proposed in (Zhou, Gandhi, Suri \& Zheng 2008) and (Jia, Zhang, Zhang \& Liu 2009a), social welfare is traded for low complexity using greedy algorithms while maintaining that truth-telling is still a dominant strategy. In (Zhou \& Zheng 2009) double auctions have been proposed for selling spectrum in the presence of multiple competitive sellers. These auctions result in truth-telling equilibria while enabling spectrum reuse to improve spectrum utilization. In (Fattahi, Fu, Van Der Schaar \& Paganini 2007), a spectrum allocation mechanism for multimedia transmission is presented. In this work users' and system performance are quantified when wireless stations employ different cross-layer strategies, and they are compared with optimal global performance. In (Wang, Wu, Ji, Liu \& Clancy 2008) a Bayesian mechanism design approach is explored for spectrum sharing based on users' channel state information.

A common feature of all of above approaches is that they are all based on direct mechanisms where the message space of the users is the same as their environment space. In contrast the approach presented in this section focussed on indirect mechanisms where the dimension of the users' message space is much smaller than that of their environment space. Furthermore, most of the above auction-based works investigate truthful implementation where social welfare is maximized at the truthful equilibrium without any guarantees about non truthful equilibria. The presentation in this section aimed at introducing a contrasting Nash implementation approach which guarantees either maximization of social welfare (Section 3.4) or attainment of Pareto optimal allocations (Section 3.5) at all Nash equilibria.

In contrast to the mechanism design approaches which construct a game form to achieve certain desirable objectives, game theoretic approaches investigate the effects of users' strategic behavior in various spectrum sharing models. Most of the game theoretic works on spectrum sharing have investigated scenarios with non-cooperative selfish users, and with $\mathrm{NE}$ as a solution concept. In (Neel, Buehrer, Reed \& Gilles 2002, Bloem, Alpcan \& Basar 2007, Tekin, Liu, South- 
well, Huang \& Ahmad n.d.) the spectrum sharing game is modeled as a noncooperative potential game (potential games were first studied in (Monderer \& Shapley 1996)) where all network users improve their strategies sequentially and converge to a Nash equilibrium in finite number of steps. Other references on potential games for spectrum sharing can be found in (Wang, Wu \& Liu 2010). Spectrum sharing problems have also been investigated within the context of evolutionary game theory (Wang, Liu \& Clancy 2010, Perez-Guirao, Luebben, Kaiser \& Jobmann 2008). In (Wang, Liu \& Clancy 2010) an evolutionary game model for cooperative spectrum sensing is proposed, and the behavior dynamics of the secondary users are studied using replicator dynamic equations. Another evolutionary game theoretic approach to cognitive radio networking is considered in (Perez-Guirao et al. 2008) where sensor nodes act as players and interact in randomly drawn pairs in an impulse radio Ultra Wide Band (UWB) sensor network. It is shown that through the interaction-learning process, a certain Quality of Service (QoS) can be guaranteed. In (Saraydar, Mandayam \& Goodman 2002, Altman \& Altman 2003, Huang et al. 2006) super-modular games have been used to design various power control algorithms arising in wireless networks. A key feature of super-modular games is strategic complementarity - if a player chooses a higher action, the others want to do the same. Strategic complementarity ensures that best response algorithms converge to NE.

A detailed discussion on the above approaches along with additional references appear in (Liu \& Wang 2011).

\section{Revenue maximization}

The previous sections considered power allocation and spectrum sharing problems where the goal correspondence is either the social welfare maximizing correspondence or the Pareto correspondence. However, if the owner of the spectrum resource is a selfish agent, it may wish to find power and spectrum allocations for the spectrum users that maximize its revenue. In this section, we look at the problem of revenue maximization. We will consider a system model less general than those discussed earlier. In particular, we will restrict ourselves to Bayesian models with utility functions that have a linear dependence on the users' types.

\section{The Model}

The general setup for power and spectrum allocation problems in this section consists of:

- A Primary Spectrum User: A primary owner of the spectrum who wants to share the channel with secondary users and collect revenue from the secondary users. Depending on the operational constraints, the primary user may need 
to allocate distinct portions of spectrum to secondary users or it may need to decide transmission power levels for the secondary users. We will refer to both spectrum and power as the allocated resource.

- Secondary Users: There are $N$ secondary users. The secondary users use the allocated spectrum/power for their communication purposes. We assume that each secondary user's utility from an allocation $x$-where $x$ may describe the allocated portions of the spectrum to users or assigned power levels to users - is characterized by its type $\theta_{i}$. For each user $i, \theta_{i} \in \Theta_{i}:=\left[\theta_{i}^{\min }, \theta_{i}^{\max }\right]$ is a random variable which is user $i$ 's private information, that is, only user $i$ knows the true value of its type. Further, each user's type is independent of other users' types. If user $i$ has type $\theta_{i}$, its utility from the allocation $x$ and paying $t_{i}$ amount of money is given as:

$$
u_{i}\left(x, t_{i}, \theta_{i}\right)=\theta_{i} \psi_{i}(x)-t_{i}
$$

The sets $\Theta_{i}$ and the function $\psi_{i}(\cdot)$ are common knowledge among the primary and secondary users. We define $\theta:=\left(\theta_{1}, \theta_{2}, \ldots, \theta_{N}\right)$ and $\Theta:=$ $\times_{i=1}^{N}\left[\theta_{i}^{\min }, \theta_{i}^{\max }\right]$.

- The Probability Distributions: All users have a strictly positive prior probability density function $f_{i}(\cdot)$ on $\theta_{i}$, with $F_{i}(\cdot)$ as the corresponding cumulative distribution function. These probability densities are common knowledge. We define $f(\theta):=\prod_{i=1}^{N} f_{i}\left(\theta_{i}\right)$ and $f_{-i}\left(\theta_{-i}\right):=\prod_{j \neq i} f_{j}\left(\theta_{j}\right)$. Because of independence of users' types the joint probability density function of all types is given by $f(\theta)$ and the joint probability density function of types of all users except the $i$ th user is given by $f_{-i}\left(\theta_{-i}\right)$.

The primary user wishes to allocate the spectrum/power in order to maximize the sum of payments $\sum_{i=1}^{N} t_{i}$ from secondary users.

In order to view the primary spectrum owner's problem from the perspective of implementation theory, we will describe it in terms of implementation theoretic concepts discussed in Section 2:

1 The primary spectrum owner is the mechanism designer who has to design a mechanism/game form that the secondary users will participate in.

2 Since the secondary users' utilities are completely characterized by their types, the environment for this problem consists only of the secondary user's types. Thus, $\mathcal{E}=\Theta$.

3 The outcome space $\mathcal{A}$ is the product space of feasible resource allocations and the payments for the secondary users. Thus, $\mathcal{A}=\mathcal{S} \times \mathbb{R}^{N}$, where $S \subset \mathbb{R}^{N}$ is the space of feasible resource allocations.

4 The goal correspondence $\gamma: \Theta \mapsto \mathcal{S} \times \mathbb{R}^{N}$ that the primary user would like to implement can be described as follows: for each $\theta \in \Theta, \gamma(\theta)$ is the set of solutions of the following optimization problem: 


$$
\begin{array}{r}
\max _{x \in \mathcal{S}, t_{i}} \sum_{i=1}^{N} t_{i} \\
\text { subject to } \theta_{i} \psi_{i}(x) \geq t_{i}
\end{array}
$$

5 Given that the secondary users only have probabilistic information about other users, we will employ the solution concept of Bayesian Nash implementation. Further, we will restrict to direct revelation mechanisms and use the less stringent requirement of truthful implementation. That is, we will only require that truthful revelation of the users' types is a Bayesian Nash equilibrium of the game and that the resulting allocations and payments at the truthful equilibrium coincide with the goal correspondence $\gamma$.

\section{Impossibility Result from Implementation Theory}

As discussed in Section 2, a necessary condition for a goal correspondence to be truthfully implementable in Bayesian Nash equilibrium is Bayesian incentive compatibility of the goal correspondence. However, it can be shown that the above correspondence $\gamma$ is not Bayesian incentive compatible. Under $\gamma$, each user should be charged a tax equal to $\theta_{i} \psi_{i}(x)$ and hence user $i$ 's net utility, $\theta_{i} \psi(x)-t_{i}=0$. If the user $i$ reports its type to be $\theta_{i}^{\prime}<\theta_{i}$, then it will be charge a tax of $\theta_{i}^{\prime} \psi_{i}(x)$ and hence user $i$ 's net utility, $\theta_{i} \psi(x)-t_{i}>0$. Therefore, $\gamma$ is not incentive compatible. Hence, no direct revelation mechanism can implement the above goal correspondence. Further, this negative result is not restricted to direct mechanisms. In absence of incentive compatibility of $\gamma$, no game form can achieve Bayesian Nash implementation of $\gamma$.

In view of the above impossibility result, the primary spectrum owner cannot hope to implement the correspondence $\gamma$ by using a direct revelation mechanism. The next pertinent question then is the following: What is the maximum expected revenue that the primary spectrum owner can achieve using a direct revelation mechanism and what is the exact structure of an expected revenue maximizing mechanism? In the rest of this section, we will present an answer to this question for two kinds of resource allocation scenarios that the primary owner may face.

\subsection{Purely Spectrum Allocation Problem}

In this Section, we consider the case where a primary spectrum user (seller) who owns $\xi$ frequency bands wants to allocate them to $N$ potential secondary users (buyers). We assume that all frequency bands are identical for the purposes of communication, that is, they provide the same bandwidth of $W \mathrm{~Hz}$ and, for each user $i$, the channel gain in all frequency bands is $h_{i i}$. If user $i$ is allocated $x_{i}$ 
number of frequency bands, where $x_{i} \in\{0,1,2, \ldots, \xi\}$, then it can achieve a rate

$$
\psi_{i}\left(x_{i}\right)=W x_{i} \log \left(1+\frac{h_{i i} P}{N_{0} W x_{i}}\right),
$$

where $P$ is the transmission power and $N_{0}$ is noise spectral density. We assume that $\psi_{i}(\cdot)$ is common knowledge among the primary and secondary users.

Note that $\psi_{i}(0):=0$ and $\psi_{i}(\cdot)$ has non-increasing increments, that is, $\psi_{i}(j+$ $1)-\psi_{i}(j) \leq \psi_{i}(j)-\psi_{i}(j-1)$, for $j=1,2, \ldots, \xi-1$. The non-increasing increment property of $\psi_{i}(\cdot)$ will be useful in later analysis.

If user $i$ has type $\theta_{i}$, its utility from the spectrum allocation and paying $t_{i}$ amount of money is given as:

$$
u_{i}\left(x_{i}, t_{i}, \theta_{i}\right)=\theta_{i} \psi_{i}\left(x_{i}\right)-t_{i}
$$

We can interpret $\theta_{i}$ as user i's "willingness to pay" - it is the maximum price per unit of rate that the user is willing to pay.

We now describe the class of mechanisms $(\mathcal{M}, h)$ we will consider. Since we will consider only direct revelation mechanisms, the message space for the $i$ th user will simply be the set of its possible types, that is, $\mathcal{M}_{i}=\Theta_{i}$. The outcome space is the product space of feasible resource allocations and the payments for the secondary users. Thus, the outcome space is $\mathcal{S} \times \mathbb{R}^{N}$, where $\mathcal{S}$ is the set of all non-negative integer valued $N$ dimensional vectors $x=\left(x_{1}, \ldots, x_{N}\right)$ with $\sum_{i=1}^{N} x_{i} \leq \xi$. Consequently, the outcome function consists of an allocation rule $q=\left(q_{1}, q_{2}, \cdots, q_{N}\right)$

$$
\begin{aligned}
& q_{i}: \Theta \rightarrow\{0,1,2, \ldots, \xi\} \text { for } i=1,2, \cdots, N, \\
& \text { such that } \sum_{i=1}^{N} q_{i}(\cdot) \leq \xi
\end{aligned}
$$

and a payment rule $t=\left(t_{1}, t_{2}, \cdots, t_{N}\right)$,

$$
t_{i}: \Theta \rightarrow \mathbb{R} \text { for } i=1,2, \cdots, N
$$

The primary user asks the secondary users to report their types. If the type vector reported is $\theta, q_{i}(\theta)$ is the amount of spectrum given to user $i$ and $t_{i}(\theta)$ is the payment charged to user $i$.

Once the mechanism $(\Theta, q, t)$ has been announced, it induces a Bayesian game among the users. Each user observes its own type but has only a probability distribution on other players' types. A user can report any type (not necessarily its true type) if it expects a higher utility by mis-reporting.

\subsubsection{Bayesian Incentive Compatibility and Voluntary Participation}

1) Bayesian Incentive Compatibility: A mechanism is Bayesian incentive compatible if truth-telling is an equilibrium of the Bayesian game induced by the mechanism. That is, each user prefers truthful reporting to any other strategy 
given that all other users are truthful. Thus, the mechanism $(q, t)$ is incentive compatible if for each $i \in \mathcal{N}$ and $\theta_{i} \in \Theta_{i}$, we have

$\mathbb{E}_{\theta_{-i}}\left[\theta_{i} \psi_{i}\left(q_{i}(\theta)\right)-t_{i}(\theta)\right] \geq \mathbb{E}_{\theta_{-i}}\left[\theta_{i} \psi_{i}\left(q_{i}\left(r_{i}, \theta_{-i}\right)\right)-t_{i}\left(r_{i}, \theta_{-i}\right)\right] \quad \forall r_{i} \in \Theta_{i}$.

2) Voluntary Participation: We will impose a participation constraint that states that each user, after learning its type, expects to get a non-negative utility by participating in the mechanism. That is, for each $i \in \mathcal{N}$ and $\theta_{i} \in \Theta_{i}$, we have

$$
\mathbb{E}_{\theta_{-i}}\left[\theta_{i} \psi_{i}\left(q_{i}(\theta)\right)-t_{i}(\theta)\right] \geq 0 .
$$

\subsubsection{The Revenue Maximization Problem}

We have the following problem for the seller.

PROBLEM 1 The sellers's optimization problem is to choose a feasible mechanism $(q, t)$ that satisfies equations (0.27) and (0.28) and maximizes its expected revenue given as:

$$
\mathbb{E}_{\theta}\left\{\sum_{i=1}^{N} t_{i}(\theta)\right\}
$$

\subsubsection{Characterizing Incentive Compatibility and Voluntary \\ Participation}

In order to solve the revenue maximization problem, we need a characterization of incentive compatibility and voluntary participation. Let $(q, t)$ be any feasible mechanism selected by the seller. In order to characterize incentive compatibility and voluntary participation for user $i$, we will adopt user $i$ 's perspective. We define the following functions for user $i$ :

DEFInition 5 Given a mechanism $(q, t)$, we define for each $\theta_{i}, r_{i} \in \Theta_{i}$,

$$
\begin{aligned}
Q_{i}\left(r_{i}\right) & :=\mathbb{E}_{\theta_{-i}}\left[\psi_{i}\left(q_{i}\left(r_{i}, \theta_{-i}\right)\right)\right], \\
T_{i}\left(r_{i}\right) & :=\mathbb{E}_{\theta_{-i}}\left[t_{i}\left(r_{i}, \theta_{-i}\right)\right], \\
U_{i}\left(\theta_{i}, r_{i}\right) & :=\theta_{i} Q_{i}\left(r_{i}\right)-T_{i}\left(r_{i}\right) .
\end{aligned}
$$

$Q_{i}\left(r_{i}\right)$ is the expected rate under the given mechanism that user $i$ will get if it reports $r_{i}$ while all other users report truthfully. Note that the expectation is over the type of all other users $\theta_{-i}$. Similarly, $T_{i}\left(r_{i}\right)$ is the expected payment that user $i$ will pay when it reports $r_{i}$ and all other users report truthfully. Also, $U_{i}\left(\theta_{i}, r_{i}\right)$ is the expected utility for user $i$ if its type is $\theta_{i}$ and it reports $r_{i}$. We can re-write the incentive compatibility and voluntary participation constraints for user $i$ in terms of the functions defined above.

Bayesian Incentive Compatibility for user $i$ :

$$
\begin{aligned}
U_{i}\left(\theta_{i}, \theta_{i}\right) & \geq U_{i}\left(\theta_{i}, r_{i}\right), \theta_{i}, r_{i} \in \Theta_{i} \\
\Longleftrightarrow \theta_{i} Q_{i}\left(\theta_{i}\right)-T_{i}\left(\theta_{i}\right) & \geq \theta_{i} Q_{i}\left(r_{i}\right)-T_{i}\left(r_{i}\right), \quad \theta_{i}, r_{i} \in \Theta_{i}
\end{aligned}
$$


Voluntary Participation for user $i$ :

$$
U_{i}\left(\theta_{i}, \theta_{i}\right) \geq 0, \theta_{i} \in \Theta_{i} \Longleftrightarrow \theta_{i} Q_{i}\left(\theta_{i}\right)-T_{i}\left(\theta_{i}\right) \geq 0, \theta_{i} \in \Theta_{i}
$$

With the above definitions of $Q_{i}(\cdot)$ and $T_{i}(\cdot)$, a user's utility $\left(\theta_{i} Q_{i}(\cdot)-T_{i}(\cdot)\right)$ is of the same form as in the optimal auction problem studied in (Myerson 1981). This allows us to find the following characterization of incentive compatible and voluntary participation using arguments similar to those in (Myerson 1981).

THEOREM 6 A mechanism $(q, t)$ satisfies Bayesian incentive compatibility and voluntary participation conditions if and only if $Q_{i}\left(r_{i}\right)$ is non-decreasing in $r_{i}$ and

$$
T_{i}\left(r_{i}\right)=K_{i}+r_{i} Q_{i}\left(r_{i}\right)-\int_{\theta_{i}^{\text {min }}}^{r_{i}} Q_{i}(s) d s,
$$

where $K_{i}=\left(T_{i}\left(\theta_{i}^{\text {min }}\right)-\theta_{i}^{\min } Q_{i}\left(\theta_{i}^{\text {min }}\right)\right) \leq 0$.

\subsubsection{An Equivalent Optimization Problem}

The primary user's objective can now be written as:

$$
\sum_{i=1}^{N} \mathbb{E}_{\theta}\left\{t_{i}(\theta)\right\}=\sum_{i=1}^{N} \mathbb{E}_{\theta_{i}}\left[\mathbb{E}_{\theta_{-i}} t\left(\theta_{i}, \theta_{-i}\right)\right]=\sum_{i=1}^{N} \mathbb{E}_{\theta_{i}}\left[T_{i}\left(\theta_{i}\right)\right]
$$

Further, because of Theorem 6 , we can write each term in the summation in $(0.33)$ as

$$
\begin{aligned}
& \mathbb{E}_{\theta_{i}}\left[T_{i}\left(\theta_{i}\right)\right]=\mathbb{E}_{\theta_{i}}\left[K_{i}+\theta_{i} Q_{i}\left(\theta_{i}\right)-\int_{\theta_{i}^{\text {min }}}^{\theta_{i}} Q_{i}(s) d s\right] \\
& =K_{i}+\int_{\theta_{i}^{\text {min }}}^{\theta_{i}^{\max }}\left[\theta_{i} Q_{i}\left(\theta_{i}\right)-\int_{\theta_{i}^{\text {min }}}^{\theta_{i}} Q_{i}(s) d s\right] f_{i}\left(\theta_{i}\right) d \theta_{i}
\end{aligned}
$$

The expression in (0.34) can be further simplified to

$$
K_{i}+\int_{\Theta}\left[\psi_{i}\left(q_{i}(\theta)\right)\left(\theta_{i}-\frac{1-F_{i}\left(\theta_{i}\right)}{f_{i}\left(\theta_{i}\right)}\right)\right] f(\theta) d \theta
$$

In the economics literature the term $\left(\theta_{i}-\frac{1-F_{i}\left(\theta_{i}\right)}{f_{i}\left(\theta_{i}\right)}\right)$ appearing in the integral in (0.35) is called virtual type.

Thus, the total expected revenue is

$$
\sum_{i=1}^{N} K_{i}+\sum_{i=1}^{N} \int_{\Theta}\left[\psi_{i}\left(q_{i}(\theta)\right)\left(\theta_{i}-\frac{1-F_{i}\left(\theta_{i}\right)}{f_{i}\left(\theta_{i}\right)}\right)\right] f(\theta) d \theta
$$

A feasible mechanism $(q, t)$ for which $K_{i}=0, i \in \mathcal{N}$ (recall that $K_{i} \leq 0$ ) and which maximizes

$$
\sum_{i=1}^{N} \int_{\Theta}\left[\psi_{i}\left(q_{i}(\theta)\right)\left(\theta_{i}-\frac{1-F_{i}\left(\theta_{i}\right)}{f_{i}\left(\theta_{i}\right)}\right)\right] f(\theta) d \theta
$$


while satisfying the conditions of Theorem 6 will be the desired revenue-maximizing mechanism.

\subsubsection{A Regularity Condition and A Candidate Solution}

We impose the following assumption on the virtual type of each user which is often called regularity condition.

ASSUMPTION 10 For each user $i,\left(\theta_{i}-\frac{1-F_{i}\left(\theta_{i}\right)}{f_{i}\left(\theta_{i}\right)}\right)$ is increasing in $\theta_{i}$.

This assumption is satisfied if $\frac{f_{i}(\theta)}{1-F_{i}(\theta)}$ is increasing in $\theta$ (that is, $f_{i}$ has an increasing hazard rate). For instance, the uniform distribution satisfies the assumption.

We can now propose a candidate solution for the seller.

TheOREm 7 For each $\theta \in \Theta$, let $q_{i}(\theta), i=1,2, \ldots, N$ be the solution of the following optimization problem:

$$
\begin{array}{cl}
\underset{x}{\arg \max } & \sum_{i=1}^{N}\left\{\psi_{i}\left(x_{i}\right)\left(\theta_{i}-\frac{1-F_{i}\left(\theta_{i}\right)}{f_{i}\left(\theta_{i}\right)}\right)\right\} \\
\text { subject to } & x_{i} \in\{0,1, \ldots, \xi\} \\
& \sum_{i=1}^{N} x_{i} \leq \xi .
\end{array}
$$

and let $t_{i}(\theta), i=1,2, \ldots, N$ be given as:

$$
t_{i}(\theta)=\theta_{i} \psi_{i}\left(q_{i}(\theta)\right)-\int_{\theta_{i}^{\text {min }}}^{\theta_{i}} \psi_{i}\left(q_{i}\left(s, \theta_{-i}\right)\right) d s .
$$

Then, $(q, t)$ maximizes the seller's expected revenue while satisfying the Bayesian incentive compatibility and voluntary participation conditions.

\subsubsection{Solving the Optimization Problem of Theorem 7}

The primary spectrum user's optimization problem in Theorem 7 has a simple intuitive solution that can be described as follows:

- Firstly, no frequency bands should be allocated to user $i$ if its virtual type is less than 0 . Since we assumed that the virtual type is an increasing function of $\theta_{i}$, there is a threshold value of $\theta_{i}$ below which the virtual type is negative. We denote this threshold value by $\theta_{i}^{t h r}$. For example, in case of $\theta_{i}$ uniformly distributed over $[0,1]$, the virtual type is negative if and only if $\theta_{i}<1 / 2$. Thus, for each user, there is a minimum threshold that the type must exceed to get any frequency band. If all users report a type less than their threshold values, no frequency band is allocated to any user.

- If only 1 user reports a type above its threshold, it is clear that all frequency bands should be allocated to that user. 
- Now assume that at least two users report types above their thresholds. We now have to solve an optimization problem. We can write the objective function as

$$
\sum_{i=1}^{N} \psi_{i}\left(x_{i}\right) w_{i}
$$

where

$$
w_{i}=\left(\theta_{i}-\frac{1-F_{i}\left(\theta_{i}\right)}{f_{i}\left(\theta_{i}\right)}\right)
$$

Further, the objective function can be written as

$$
\begin{aligned}
& \sum_{i=1}^{N} w_{i} \sum_{j=1}^{x_{i}}\left\{\psi_{i}(j)-\psi_{i}(j-1)\right\} \\
& =\sum_{i=1}^{N} \sum_{j=1}^{x_{i}} w_{i}\left\{\psi_{i}(j)-\psi_{i}(j-1)\right\}
\end{aligned}
$$

$w_{i}\left(\psi_{i}(j)-\psi_{i}(j-1)\right)$ can be thought of as "virtual marginal utility" of user $i$ if it already has $j-1$ frequency bands. If we consider the set of all possible virtual marginal utilities corresponding to the reported types:

$$
\mathbb{M}=\left\{w_{i}\left(\psi_{i}(j)-\psi_{i}(j-1)\right), i=1,2, \ldots, N ; j=1,2, \ldots, \xi\right\},
$$

then each term in equation $(0.41)$ is a distinct term from the set $\mathrm{M}$. Moreover, there are no more than $\xi$ terms in (0.41) since $\sum_{i=1}^{N} x_{i} \leq \xi$. This means the objective function can be no larger than the sum of $\xi$ largest terms of the set $\mathbb{M}$. There is a clear way of choosing allocations so that the objective function is equal to the sum of $\xi$ largest terms of IM: Give the first channel to user corresponding to the largest term in $\mathrm{M}$, the second channel to the user corresponding to the second largest term in $\mathrm{M}$ and so on. Thus, we have a simple description of the optimal solution of the primary user's optimization problem: the frequency bands are allocated sequentially such that at each stage the user with the highest virtual marginal utility gets the next channel.

The tax function is given by the equation:

$$
t_{i}(\theta)=\theta_{i} \psi_{i}\left(q_{i}(\theta)\right)-\int_{\theta_{i}^{\text {min }}}^{\theta_{i}} \psi_{i}\left(q_{i}\left(s, \theta_{-i}\right)\right) d s .
$$

The tax can also be described in an intuitive way:

- Suppose that for a given profile of types, user $i$ does not get any frequency bands, that is, $q_{i}(\theta)=0$. Then, it can be shown that $q_{i}\left(s, \theta_{-i}\right)=0$, for all $s<\theta_{i}$ which implies $\int_{\theta_{i}^{\text {min }}}^{\theta_{i}} \psi_{i}\left(q_{i}\left(s, \theta_{-i}\right)\right) d s=\int_{\theta_{i}^{m i n}}^{\theta_{i}} \psi_{i}(0) d s=0$. Thus, if a user $i$ does not get any frequency band, its tax is 0 . 
- Suppose user $i$ is the only user whose type exceeds its threshold. Then, user $i$ get all the bands. Then,

$$
\begin{aligned}
t_{i}(\theta) & =\theta_{i} \psi_{i}(\xi)-\int_{\theta_{i}^{\text {min }}}^{\theta_{i}} \psi_{i}\left(q_{i}\left(s, \theta_{-i}\right)\right) d s \\
& =\theta_{i} \psi_{i}(\xi)-\left\{\int_{\theta_{i}^{\text {min }}}^{\theta_{i}^{t h r}} \psi_{i}\left(q_{i}\left(s, \theta_{-i}\right)\right) d s+\int_{\theta_{i}^{t h r}}^{\theta_{i}} \psi_{i}\left(q_{i}\left(s, \theta_{-i}\right)\right) d s\right\} \\
& =\theta_{i} \psi_{i}(\xi)-\left\{\int_{\theta_{i}^{\text {min }}}^{\theta_{i}^{t h r}} \psi_{i}(0) d s+\int_{\theta_{i}^{t h r}}^{\theta_{i}} \psi_{i}(\xi) d s\right\} \\
& =\theta_{i} \psi_{i}(\xi)-\left\{0+\theta_{i} \psi_{i}(\xi)-\theta_{i}^{t h r} \psi_{i}(\xi)\right\} \\
& =\theta_{i}^{t h r} \psi_{i}(\xi)
\end{aligned}
$$

Thus, when all the other users have 0 allocations, the price per unit rate that the user $i$ pays is the minimum type that would have given it all the bands.

- Finally, consider the case when more than 1 user has a non-zero allocation. Let $\mathbb{M}_{-i}$ be the set of virtual marginal utilities of all users except $i$.

$$
\mathbb{M}_{-i}=\left\{w_{k}\left(\psi_{k}(j)-\psi_{k}(j-1)\right), k=1,2, \ldots, N, k \neq i ; j=1,2, \ldots, \xi\right\},
$$

and $\mathbb{I M}_{i}$ be the set of virtual marginal utilities of user $i$.

$$
\mathbf{M}_{i}=\left\{w_{i}\left(\psi_{i}(j)-\psi_{i}(j-1)\right), j=1,2, \ldots, \xi\right\},
$$

Given the set $\mathbb{M}_{-i}$, we can define

$$
\begin{gathered}
\rho_{i}^{j}:=\min \left\{\theta_{i}: \text { exactly } j \text { largest terms of } \mathbb{M}_{i} \text { exceed the }(\xi-j+1)\right. \text { th } \\
\text { largest term of } \left.\mathbb{M}_{-i}\right\}
\end{gathered}
$$

Then, using arguments similar to those in earlier cases, we can show that if user $i$ gets $x_{i}$ bands, the total tax paid by user $i$ is $\sum_{j=1}^{x_{i}} \rho_{i}^{j}\left(\psi_{i}(j)-\psi_{i}(j-1)\right)$. For example, if $x_{i}=1$,

$$
\begin{aligned}
t_{i}(\theta)=\theta_{i} \psi_{i}(1)-\int_{\theta_{i}^{\text {min }}}^{\theta_{i}} \psi_{i}\left(q_{i}\left(s, \theta_{-i}\right)\right) d s \\
=\theta_{i} \psi_{i}(1)-\left\{\int_{\theta_{i}^{\text {min }}}^{\rho_{i}^{1}} \psi_{i}\left(q_{i}\left(s, \theta_{-i}\right)\right) d s+\int_{\rho_{i}^{1}}^{\theta_{i}} \psi_{i}\left(q_{i}\left(s, \theta_{-i}\right)\right) d s\right\} \\
=\theta_{i} \psi_{i}(1)-\left\{\int_{\theta_{i}^{\text {min }}}^{\rho_{i}^{1}} \psi_{i}(0) d s+\int_{\rho_{i}^{1}}^{\theta_{i}} \psi_{i}(1) d s\right\} \\
=\theta_{i} \psi_{i}(1)-\left\{0+\theta_{i} \psi_{i}(1)-\rho_{i}^{1} \psi_{i}(1)\right\} \\
=\rho_{i}^{1} \psi_{i}(1)
\end{aligned}
$$




$$
\begin{aligned}
& \text { If } x_{i}=2, \\
& t_{i}(\theta)=\theta_{i} \psi_{i}(2)-\int_{\theta_{i}^{\text {min }}}^{\theta_{i}} \psi_{i}\left(q_{i}\left(s, \theta_{-i}\right)\right) d s \\
& =\theta_{i} \psi_{i}(2)-\left\{\int_{\theta_{i}^{\text {min }}}^{\rho_{i}^{1}} \psi_{i}\left(q_{i}\left(s, \theta_{-i}\right)\right) d s+\int_{\rho_{i}^{1}}^{\rho_{i}^{2}} \psi_{i}\left(q_{i}\left(s, \theta_{-i}\right)\right) d s+\int_{\rho_{i}^{2}}^{\theta_{i}} \psi_{i}\left(q_{i}\left(s, \theta_{-i}\right)\right) d s\right\} \\
& =\theta_{i} \psi_{i}(2)-\left\{0+\rho_{i}^{2} \psi_{i}(1)-\rho_{i}^{1} \psi_{i}(1)+\theta_{i} \psi_{i}(2)-r_{i}^{2} \psi_{i}(2)\right\} \\
& =\rho_{i}^{2}\left(\psi_{i}(2)-\psi_{i}(1)\right)+\rho_{i}^{1}\left(\psi_{i}(1)-\psi_{i}(0)\right) \\
& =\sum_{j=1}^{2} \rho_{i}^{j}\left(\psi_{i}(j)-\psi_{i}(j-1)\right)
\end{aligned}
$$

\subsection{Purely Power Allocation Problem}

The above approach for finding revenue maximizing allocations can also be applied to the situation where the primary user wishes to allocate transmission power levels to the secondary users. In this setup, all users can transmit over the entire spectrum at their assigned power levels. This creates interference among secondary users as well as among the secondary and primary users. Assuming that the primary user can tolerate interference from the secondary users as long as the total transmission power of all secondary users is below $P$, the primary user's problem is to design an allocation and payment mechanism to maximize its expected revenue. It is possible to extend the analysis of the previous section to characterize the optimal mechanism for this case as well. However, the presence of interference among users implies that optimal allocations are solution of a non-convex problem and thus more difficult to compute. We refer the reader to (Kakhbod, Nayyar \& Teneketzis 2011) for details.

\subsection{Other Models and Approaches on Revenue Maximization}

For the problem of expected revenue maximization for the primary user, we have taken the approach used in (Myerson 1981) for a single object auction. According to this approach, once the primary user announces its allocation and payment rule, the secondary users decide whether to participate in the mechanism and if they do participate, they play a Bayesian game. Such an approach implies that we must find revenue-maximizing mechanism within the class of mechanisms that satisfy Bayesian incentive compatibility and voluntary participation constraints. A similar viewpoint has been adopted in (Jia, Zhang, Zhang \& Liu 2009b) for a related problem of finding revenue maximizing spectrum allocations with interference constraints where each user either gets all the channels it asked for or gets nothing. The work in (Ledyard 2007) provides a revenue-maximizing auction where each user's utility depends only on whether it is allocated a particular subset of the goods (channels) being auctioned. Once again, the auction is re- 
quired to satisfy Bayesian incentive compatibility and voluntary participation constraints.

Several other works have adopted a conceptually different approach by looking at the problem of maximizing revenue without dealing with incentive compatibility and voluntary participation issues. For example, in (Kasbekar \& Sarkar 2010), it is assumed that each user submits the amount it is willing to pay for each possible spectrum allocation. The primary user is then faced with a combinatorial optimization problem to find allocations that maximize the sum of payments. Similarly, in (Gandhi, Buragohain, Cao, Zheng \& Suri 2008), the authors consider the situation where a price - demand curve is given for each user. The goal there is to solve a combinatorial optimization problem to maximize the revenue under some interference constraints.

\section{Conclusion and reflections}

We have presented an implementation theory approach to power allocation and spectrum sharing problems that arise in wireless networks. This approach has the following desirable features: (1) It allows us to know when a decentralized resource allocation problem with strategic users admits an optimal solution, (2) In the cases where the decentralized allocation problem admits an optimal solution, it provides guidelines for the design of game forms that achieve optimal allocations (such as those in Section 3), (3) In the cases where the decentralized allocation problem does not admit an optimal solution, it suggests alternative criteria (such as those in Section 4) for the design of game forms that result in satisfactory allocations.

The game forms presented in Section 3 ensure that the desired allocations are achieved at equilibria without specifying how an equilibrium is reached. That is, the game forms in Section 3 do not include an iterative/tâtonnement process that determines how the NE of the game induced by the game form are computed by the users. The lack of such iterative processes for decentralized resource allocation problems where strategic users possess private information is a major open problem in implementation theory. The major difficulty in constructing iterative algorithms that guarantee convergence to $\mathrm{NE}$ is the following: Consider an iterative algorithm for a decentralized allocation problem where strategic users possess private information. At each stage of the algorithm each user updates its message. A user, say user $i$, can report any message it deems beneficial to itself and other users may not be able to check whether or not user $i$ is following the rules of the iterative algorithm. Consequently, the algorithm must provide incentives to the users to follow the rules at each stage of the algorithm. Such a provision of incentives must be based on all the information available at the current stage and must, in general, take the whole future into account. Algorithms with the above features are currently unavailable. The only exception is 
the work on virtual subgame perfect equilibrium implementation ((Osborne \& Rubinstein 1994, Chapter 10)).

Game theoretic approaches for decentralized resource allocation problems where the strategic users do not possess private information have provided algorithms for the computation of equilibrium (as evidenced by the discussion in section 3.7). However, most of the games presented in the literature so far possess multiple equilibria and only a subset of these equilibria result in allocations that are optimal with respect to a pre-specified performance metric.

In this chapter, we have investigated static decentralized resource allocations problems. Dynamic decentralized resource allocation problems where the number of users, or the users' utilities or the set of users with whom a particular user interacts changes over time are a class of important problems. Currently, very little is known about the nature of solution of these problems. Implementation theory has not been successful in addressing dynamic decentralized resource allocation problems. So far, work on dynamic mechanism design has addressed only truthful implementation in NE (Bergemann \& Valimaki 2010). Thus, the systematic design of dynamic power allocation and spectrum sharing mechanisms that implement social choice correspondences such as the welfare maximizing correspondence while satisfying individual rationality and budget balance conditions is currently an important open problem. 


\section{References}

Altman, E. \& Altman, Z. (2003), 'S-modular games and power control in wireless networks', IEEE Transactions on Automatic Control 48, 839-842.

Bajari, P. \& Yeo, J. (2009), 'Auction design and tacit collusion in FCC spectrum auctions', Information Economics and Policy 22(2), 90-100.

Bergemann, D. \& Valimaki, J. (2010), 'The dynamic pivot mechanism', Econometrica 78(2), 771-689.

Bloem, M., Alpcan, T. \& Basar, T. (2007), A Stackelberg game for power control and channel allocation in cognitive radio networks, in 'ValueTools '07 Proceedings of the 2nd international conference on Performance evaluation methodologies and tools', number 4 .

Chen, Y., Yu, G., Zhang, Z., Chen, H. \& Qiu, P. (2008), 'On cognitive radio networks with opportunistic power control strategies in fading channels', IEEE Transactions on Wireless Communications 7(7), 2752-2761.

Dasgupta, P., Hammonds, P. \& Maskin, E. (1979), 'The implementation of social choice rules: Some general results on incentive compatibility', The Review of Economic Studies 46, $185-216$.

Fattahi, A., Fu, F., Van Der Schaar, M. \& Paganini, F. (2007), 'Mechanism-based resource allocation for multimedia transmission over spectrum agile wireless networks', IEEE Journal on Selected Areas in Communications 25(3), 601-612.

Gandhi, S., Buragohain, C., Cao, L., Zheng, H. \& Suri, S. (2008), 'Towards real-time dynamic spectrum auctions', Computer Networks 52(4), 879 - 897.

Green, J. \& Laffont, J. (1979), Incentives in Public Decision Making, Ameterdam, North Holland.

Groves, T. \& Ledyard, J. (1987), Incentive compatibility since 1972, In T. Groves, R. Radner and S. Reiter, editors, Information, Incentives, and Economic Mechanisms: Essays in Honor of Leonid Hurwicz, University of Minnesota Press, Minneapolis, pp. 48-109.

Huang, J., Berry, R. A. \& Honig, M. L. (2006), 'Auction-based spectrum sharing', ACM/Springer Mobile Networks and Applications Journal (MONET) 11(3), 405418.

Huang, J., Berry, R. \& Honig, M. (2004), Auction mechanisms for distributed spectrum sharing, in '42nd Allerton Conference on Communication, Control, and Computing'.

Hurwicz, L. (1979), 'Outcome functions yielding Walrasian and Lindahl allocations at Nash equilibrium points', Review of Economic studies 46, 217-225.

Hurwicz, L., Maskin, E. \& Postlewaite, A. (1995), Feasible nash implementation of social choice correspondence when the designer does not know endowments or pro- 
duction sets, In J. Ledyard, editor, The Economics of Information Decentralization: Complexity, Efficiency and Stability, Klewer, Amesterdam.

J. Nash (1950), Non-Cooperative Games, PhD thesis, Princeton University.

Jackson, M. (2001), A crash course in implementation theory, in 'Social choice and welfare', pp. 655-708.

Jackson, M. O. (1991), 'Bayesian implementation', Econometrica 59(1), 461-477.

Jia, J., Zhang, Q., Zhang, Q. \& Liu, M. (2009a), Revenue generation for truthful spectrum auction in dynamic spectrum access, in 'Proceedings of the 10th ACM International Symposium on Mobile Ad Hoc Networking', pp. 3-12.

Jia, J., Zhang, Q., Zhang, Q. \& Liu, M. (2009b), 'Revenue generation for truthful spectrum auction in dynamic spectrum access', In proc. of ACM International Symposium on Mobile Ad Hoc Networking and Computing (MobiHoc).

Kakhbod, A., Nayyar, A. \& Teneketzis, D. (2011), Revenue maximization in spectrum auctions for dynamic spectrum access. arXiv:1105.5743v1.

Kakhbod, A. \& Teneketzis, D. (2012a), 'An efficient game form for multi-rate multicast service provisioning', IEEE Journal on Selected Areas in Communications 30(11).

Kakhbod, A. \& Teneketzis, D. (2012b), 'An efficient game form for unicast service provisioning', IEEE Transaction on Automatic Control 57(2), 392-404.

Kakhbod, A. \& Teneketzis, D. (2012c), 'Power allocation and spectrum sharing in multiuser multi-channel systems with strategic users', IEEE Transactions on Automatic Control 57(9), 2338-2343.

Kasbekar, G. \& Sarkar, S. (2010), 'Spectrum auction framework for access allocation in cognitive radio networks', IEEE/ACM Transactions on Networking 18(6), 18411854.

Kash, I. A. \& Parkes, D. C. (2010), 'Impersonation strategies in auctions', Lecture Notes in Computer Science 6484 pp. 492-495.

Ledyard, J. O. (2007), Optimal combinatoric auctions with single-minded bidders, in 'Proceedings of the 8th ACM conference on Electronic commerce', EC '07, pp. 237242.

Liu, K. J. R. \& Wang, B. (2011), Cognitive Radio Networking and Security, A GameTheoretic View, Cambridge University Press.

Mas-Colell, A., Whinston, M. D. \& Green, J. R. (2005), Microeconomic theory, Oxford University Press.

Maskin, E. (1999), 'Nash equilibrium and welfare optimality', The Review of Economic Studies 66, 23 - 38 .

Maskin, E. (n.d.), The theory of implementation in Nash equilibrium: A survey, in L. Hurwicz, D. Schmeidler \& H. Sonnenschein, eds, 'Social goals and social organization', Cambridge university press.

Monderer, D. \& Shapley, L. (1996), 'Potential games', Games and Economic Behavior 14(1), 124-143.

Myerson, R. (1981), 'Optimal auction design', "Mathematics of Operations Research" pp. $58-73$.

Neel, J., Buehrer, R., Reed, J. \& Gilles, R. (2002), 'Game theoretic analysis of a network of cognitive radios', in: IEEE Midwest Symposium on Circuits and Systems 45.

Osborne, M. \& Rubinstein, A. (1994), A Course in Game theory, The MIT Press.

Palfrey, T. (1994), Implementation theory, In R. Aumann and S. Hart, editors, Handbook of Game Theory with Economic Applications, North, Holland. 
Palfrey, T. \& Srivastava, S. (1989), 'Implementation with incomplete information in exchange', Econometrica 57, 115-134.

Palfrey, T. \& Srivastava, S. (n.d.a), Bayesian implementation, in 'Fundamentals of Pure and Applied Economics 53', Harwood Academic.

Palfrey, T. \& Srivastava, S. (n.d.b), Bayesian implementation, in 'Fundamentals of Pure and Applied Economics 53', Harwood Academic.

Perez-Guirao, M., Luebben, R., Kaiser, T. \& Jobmann, K. (2008), 'Evolutionary game theoretical approach for ir-uwb sensor networks', in: IEEE International Conference on Communications Workshops pp. 107-111.

Reichelstein, S. \& Reiter, S. (1988), 'Game forms with minimal message space', Econometrica 56(3), 661-692.

Saraydar, C., Mandayam, N. \& Goodman, D. (2002), 'Efficient power control via pricing in wireless data networks', IEEE Transactions on Communications 50, 291-303.

Sharma, S. (2009), A mechanism design approach to decentralized resource allocation in wireless and large-scale networks: Realization and implementation, $\mathrm{PhD}$ thesis, University of Michigan, Ann Arbor.

Sharma, S. \& Teneketzis, D. (2009), 'An externalities-based decentralized optimal power allocation algorithm for wireless networks', IEEE/ACM Transactions on Networking 17(6), 1819-1831.

Sharma, S. \& Teneketzis, D. (2011), 'A game-theoretic approach to decentralized optimal power allocation for cellular networks', Telecommunication Systems journal 47, 65-80.

Sharma, S. \& Teneketzis, D. (2012), 'Local public good provisioning in networks: A Nash implementation mechanism', IEEE Journal on Selected Areas in Communication $\mathbf{3 0}(11)$.

Stoenescu, T. \& Ledyard, J. (2006), A pricing mechanism which implements in Nash equilibria a rate allocation problem in networks, in 'Decision and Control, 2006 45th IEEE Conference on', pp. $1270-1277$.

Tekin, C., Liu, M., Southwell, R., Huang, J. \& Ahmad, S. (n.d.), 'Atomic congestion games on graphs and their applications in networking', To appear in: IEEE/ACM Transactions on Networking.

Thompson, W. (2001), 'Concepts of implementation', Japanese Economic Review 47, $133-143$.

Wang, B., Liu, K. \& Clancy, T. (2010), 'Evolutionary cooperative spectrum sensing game: how to collaborate?', IEEE Transactions on Communications 58(3), 890-900.

Wang, B., Wu, Y., Ji, Z., Liu, K. \& Clancy, T. (2008), 'Game theoretical mechanism design methods: suppressing cheating in cognitive radio networks', IEEE Signal Processing Magazine 25(6), 74-84.

Wang, B., Wu, Y. \& Liu, R. (2010), 'Game theory for cognitive radio networks: An overview', Computer Networks 54, 2537-2561.

Zhou, X., Gandhi, S., Suri, S. \& Zheng, H. (2008), ebay in the sky: strategy-proof wireless spectrum auctions, in 'Proceedings of the 14th ACM International Conference on Mobile Computing and Networking', pp. 2-13.

Zhou, X. \& Zheng, H. (2009), Trust: a general framework for truthful double spectrum auctions, in 'Proceedings of the IEEE INFOCOM'. 